\title{
A Posteriori Error Estimation and Adaptive Computation of Viscoelastic Fluid Flow
}

\author{
Vincent J. Ervin* Louis N. Ntasin \\ Department of Mathematical Sciences, \\ Clemson University, \\ Clemson, South Carolina 29634-0975
}

\begin{abstract}
In this paper, we develop a posteriori error estimates for Finite Element (FE) approximations of viscoelastic fluid flows governed by differential constitutive laws of Giesekus and Oldroyd-B type. We use the general framework developed by Verfürth for constructing residual based a posteriori error estimates for nonlinear equations. Numerical experiments using adaptive computations demonstrating the effectiveness of these error estimates are then presented for three examples. The first two examples are problems with known solutions and the third example is, a benchmark problem, the channel flow with a cylindrical obstacle problem.
\end{abstract}

Key words. viscoelastic, finite element method, a posteriori error, adaptivity, SUPG.

AMS Mathematics subject classifications. 65 N30

\section{Introduction}

In the past two decades, numerical simulations of viscoelastic fluid flows governed by differential constitutive laws has been a major challenge to scientists. Coupling of the saddle point problem formed by the momentum and continuity equations, and a differential constitutive law (that is hyperbolic in character) poses significant numerical challenges. Maintaining the ellipticity of the saddle point problem over the entire domain has been a major challenge, especially within regions of high stress transitions. Another major difficulty encountered is dealing with singularities of the solution in high stress transition regions. Also, in practical applications, discretized viscoelastic flow problems yield very large linear systems. Solving such systems has also proven to be challenging due to computer processing speed and memory limitations in computers, making the solution process a bottleneck in numerical simulations. These three sources of difficulty have limited numerical

\footnotetext{
${ }^{*}$ This work was partially supported by the ERC Program of the National Science Foundation under Award Number ERC-9731680.
} 
simulations to fluids with limited elastic effects. Numerical simulation of fluids with high elastic effects has proven to be a challenge; known commonly as the "High Deborah ${ }^{1}$ number" problem (or the "High Weissenberg number" problem).

Solution schemes aimed at resolving the "High Deborah number" problem have been proposed over the past years. These include EEME [14], EVSS [22], EVSS-G [26], DEVSS-G [17], AVSS [24], and DAVSS-G [25]. These schemes have increased the Deborah number for flows that can be simulated for particular applications.

To combat stress singularities especially in the high stress transition regions, various stabilizing schemes for discretizing the constitutive equations have been studied. These include the Discontinuous Galerkin (DG) method of Lesaint and Raviart [16], Streamline Upwind (SU) method [13], and the Streamline Upwind Petrov Galerkin (SUPG) method of Brooks and Hughes [3]. Based on a "small data" assumption, SUPG and DG have been shown for problems in $\mathbb{R}^{2}$ to converge at a rate of $h^{3 / 2}[1,23]$, where $h$ is the spatial mesh size.

Within the framework of Finite Element (FE) methods, generating optimal or near optimal meshes is a useful technique for increasing accuracy at a lower computational costs. A posteriori error estimates have been used with much success as a guiding tool in adaptively generating optimal or near optimal meshes, and in adaptively computing solutions to problems with boundary layers (regions of rapid transition of the solution) (see: [7, 8, 9]). Sandri [23] derived a posteriori error estimates for a fluid obeying the power law. Recent results have also been published on a posteriori error estimates for some quasi-Newtonian Stokes flows [11] and for a nonlinear three-field problem arising from Oldroyd-B viscoelastic flows [20]. Owens [19] and Chauvière and Owens [5] have constructed error indicators for spectral element methods for an Oldroyd-B fluid.

In this paper, we develop a posteriori error estimates for the Giesekus and Oldroyd-B type differential constitutive laws. We start by defining the problem and its governing equations. An abstract framework from [27] for constructing a posteriori error estimates for non-linear differential equations is then presented. After describing some of the FE tools necessary for constructing the error estimates, viscoelastic fluid flow equations are then cast in this framework. A posteriori error estimates are then constructed. In section 5, numerical results are presented which demonstrate the effectiveness of the procedure.

\section{Problem Definition and Notation}

Consider an isothermal viscoelastic fluid flowing in a bounded, connected, open domain $\Omega \subset$ $\mathbb{R}^{n},(n=2,3)$ with Lipschitz boundary $\Gamma=\partial \Omega$. Let $\mathbf{n}$ be the unit outer normal to the boundary, $\Gamma$.

We adopt the following notation: Let $x_{i}, i=1, \ldots, n$, be cartesian coordinates in $\mathbb{R}^{n}$; given a function $u, \frac{\partial u}{\partial x_{i}}$ is written as $u,_{i}$ and the partial derivative with respect to time $\frac{\partial u}{\partial t}$ is $u_{t}$. If $p$ is a scalar function then $\nabla p$ defines the gradient of $p$ which is a vector with $(\nabla p)_{i}=p,_{i}$. If $u$ is a vector

\footnotetext{
${ }^{1}$ The Deborah number represents a measure of the ratio of the magnitude of the elastic forces to that of the viscous forces[2].
} 
then the gradient of $u, \nabla u$, is a second order tensor written as $(\nabla u)_{i, j}=u_{j, i}$, the divergence of $u$ is a scalar given as $\nabla \cdot u=u_{i, i}$, and $u \cdot \nabla=u_{i} \frac{\partial}{\partial x_{i}}$. If $\tau$ and $\sigma$ are second order tensors then the matrix product of $\tau$ and $\sigma, \tau \sigma$ is a second order tensor with $(\tau \sigma)_{i j}=\tau_{i k} \sigma_{k j}$, the divergence of $\tau, \nabla \cdot \tau$ is a vector with $(\nabla \cdot \tau)_{i}=\tau_{j i, j}$ and $\sigma: \tau=\sigma_{i j} \tau_{i j}$ is a scalar.

For viscoelastic fluid flows, the pressure $p$ is a scalar function, velocity $u$ is a vector function, while the total stress $\tau_{\text {tot }}$ is a second order tensor.

The conservation of momentum equation can be expressed as

$$
\rho u_{t}+\rho(u \cdot \nabla) u=\nabla \cdot \tau_{\text {tot }}+\rho \tilde{f},
$$

where $\tilde{f}$ is a combination of the body forces on the fluid, and $\rho$ the density of the fluid.

The total stress tensor, $\tau_{\text {tot }}$ is written as

$$
\tau_{t o t}=-p \mathbb{I}+\tau_{N}+\tau,
$$

where $\mathbb{I}$ is the unit tensor, $\tau_{N}$ is the Newtonian component of the stress and $\tau$ is the viscoelastic stress component.

For a broad class of differential constitutive models written in non-dimensional form, the viscoelastic stress component may be modeled by the following constitutive law:

$$
\lambda\left(\frac{\partial_{a} \tau}{\partial t}+\frac{\gamma}{\alpha}(\tau \tau)\right)+\tau=2 \alpha \mathbf{D}(u)
$$

where $\frac{\partial_{a} \tau}{\partial t}$ denotes an objective derivative defined in $(2.3), \mathbf{D}(u)=\frac{1}{2}\left(\nabla u+(\nabla u)^{T}\right)$ is called the rate of strain tensor (deformation tensor), and $\alpha \in(0,1)$ and $\gamma$ are model parameters.

To complete the formulation we need the conservation of mass equation

$$
\frac{\partial \rho}{\partial t}+\nabla \cdot(\rho u)=0
$$

In this study we make the following two assumptions:

A1: The flow is time independent and creeping.

A2: The fluid is incompressible.

Based on assumption A1, and the decomposition of $\tau_{\text {tot }}$ the balance of momentum equation becomes

$$
-\nabla \cdot\left(-p \mathbb{I}+\tau_{N}+\tau\right)=f
$$

while the conservation of mass equation reduces to

$$
\nabla \cdot u=0
$$


A general definition of $\frac{\partial_{a} \tau}{\partial t}$ describing a wide variety of models is given as

$$
\frac{\partial_{a} \tau}{\partial t}=\tau_{t}+(u \cdot \nabla) \tau+g_{a}(\tau, \nabla u)
$$

where

$$
g_{a}(\tau, \nabla u)=\mathbf{W}(u) \tau-\tau \mathbf{W}(u)-a(\mathbf{D}(u) \tau+\tau \mathbf{D}(u)),
$$

and $\mathbf{W}(u)=\frac{1}{2}\left(\nabla u-(\nabla u)^{T}\right)$ denotes the vorticity tensor, and $a \in[-1,1]$ is a parameter. The choices $a=1,-1,0$ corresponding to the upper, lower and corrotational convected derivatives, respectively.

The Newtonian stress $\tau_{N}$, is defined as

$$
\tau_{N}=2(1-\alpha) \mathbf{D}(u)
$$

Incorporating this definition of $\tau_{N}$ into the momentum equation we have

$$
-2(1-\alpha) \nabla \cdot \mathbf{D}(u)-\nabla \cdot \tau+\nabla p=f .
$$

Note: The case $\gamma=0$ in (2.2) yields the Oldroyd-B model.

Associated with the model equations we assume the following boundary conditions. For velocity we let $u=u_{0}$ at the boundary $\Gamma$. For the stress we specify $\tau=\tau_{0}$ at the inflow boundary $\Gamma^{-}$, where $\Gamma^{-}:=\{x \in \Gamma, \quad \mid u \cdot \mathbf{n}<0\}$. These boundary conditions are motivated by the character of the governing equations : momentum equation being elliptic while the constitutive equation is hyperbolic.

In order to simplify the analysis, take $u_{0}=0$. Then, as there is no inflow boundary, no boundary condition is necessary for the stress. Additionally, we restrict our attention to the case $\lambda>0$. For $\lambda=0$ the model may be rewritten as a Stokes problem. Thus the problem of interest can be written as:

Problem (O): Find $(u, \tau, p)$ such that

$$
\begin{aligned}
\tau+\lambda(u \cdot \nabla) \tau+\lambda \frac{\gamma}{\alpha}(\tau \tau)+\lambda g_{a}(\tau, \nabla u)-2 \alpha \mathbf{D}(u)=0 & \text { in } \Omega, \\
-2(1-\alpha) \nabla \cdot \mathbf{D}(u)-\nabla \cdot \tau+\nabla p=f & \text { in } \Omega, \\
\nabla \cdot u=0 & \text { in } \Omega, \\
u=0 & \text { on } \Gamma .
\end{aligned}
$$

\section{A Posteriori Error Estimation for Nonlinear Differential Equa- tions}

In this section we present the abstract framework as described in [27] for constructing a posteriori error estimates for non-linear differential equations, followed by a collection of Finite Element approximation tools. 


\subsection{Abstract a posteriori error estimates}

For ease of exposition we follow the notation used in Verfürth [27]. Let $X$ and $Y$ be two Banach spaces with the norms $\|\cdot\|_{X}$ and $\|\cdot\|_{Y}$ respectively. For any element $u \in X$ and any real number $R>0$ define

$$
B(u, R):=\left\{v \in X \mid\|u-v\|_{X}<R\right\} .
$$

Let $\mathcal{L}(X, Y)$ denote the Banach space of continuous linear maps from $X$ to $Y$ equipped with the operator norm $\|\cdot\|_{\mathcal{L}(X, Y)}$. Denote by $\operatorname{Isom}(X, Y) \subset \mathcal{L}(X, Y)$ the open subset of linear homeomorphisms of $X$ onto $Y$. The dual space of $Y, Y^{*}:=\mathcal{L}(Y, \mathbb{R})$ and $<\cdot, \cdot>$ represents the duality pairing between $Y$ and $Y^{*}$.

Let $F \in C^{1}\left(X, Y^{*}\right)$ be a continuously differentiable function. Denote the linearization of $F$ about $u_{0}$ by $D F\left(u_{0}\right)$.

Theorem 3.1 (Verfürth [27]) Let $u_{0} \in X$ satisfy $F\left(u_{0}\right)=0$ and assume there exists (non-trivial) subspaces $X_{D} \subset X, Y_{D}^{*} \subset Y^{*}$ such that $D F\left(u_{0}\right) \in \operatorname{Isom}\left(X_{D}, Y_{D}^{*}\right)$. In addition, assume that $D F$ is Lipschitz continuous at $u_{0}$ i.e. there is an $R_{0}>0$ such that

$$
\Upsilon:=\sup _{u \in B\left(u_{0}, R_{0}\right)} \frac{\left\|D F(u)-D F\left(u_{0}\right)\right\|_{\mathcal{L}\left(X, Y^{*}\right)}}{\left\|u-u_{0}\right\|_{X}}<\infty,
$$

and let $R_{\min }$ be given by

$$
R_{\text {min }}=\min \left\{R_{0}, \Upsilon^{-1}\left\|D F\left(u_{0}\right)^{-1}\right\|_{\mathcal{L}\left(Y_{D}^{*}, X_{D}\right)}^{-1}, 2 \Upsilon^{-1}\left\|D F\left(u_{0}\right)\right\|_{\mathcal{L}\left(X, Y^{*}\right)}\right\} .
$$

Then for any $u \in B\left(u_{0}, R_{\text {min }}\right) \cap X_{D}$ we have the estimates

$$
\frac{1}{2}\left\|D F\left(u_{0}\right)\right\|_{\mathcal{L}\left(X, Y^{*}\right)}^{-1}\|F(u)\|_{Y^{*}} \leq\left\|u-u_{0}\right\|_{X},
$$

and

$$
\left\|u-u_{0}\right\|_{X} \leq 2\left\|D F\left(u_{0}\right)^{-1}\right\|_{\mathcal{L}\left(Y_{D}^{*}, X_{D}\right)}\|F(u)\|_{Y^{*}} .
$$

This theorem provides an abstract framework on which a posteriori error estimates for non-linear differential equations can be constructed. An obvious difficulty in using this error estimator is that the true solution $u$ is not known. Generally, the multipliers $\left\|D F\left(u_{0}\right)\right\|_{\mathcal{L}\left(X, Y^{*}\right)}^{-1}$ and $\left\|D F\left(u_{0}\right)^{-1}\right\|_{\mathcal{L}\left(Y_{D}^{*}, X_{D}\right)}$ can be approximated by $\left\|D F\left(u_{h}\right)\right\|_{\mathcal{L}\left(X_{h}, Y_{h}^{*}\right)}^{-1}$ and $\left\|D F\left(u_{h}\right)^{-1}\right\|_{\mathcal{L}\left(Y_{h}^{*}, X_{h}\right)}$, respectively, given an approximate solution $u_{h}$, where $X_{h} \subset X_{D} \subset X$ and $Y_{h} \subset Y \subset Y_{D}$ are appropriate Finite Element (FE) spaces.

As noted in [27] (Remark 2.2) estimate (3.10) can be modified to obtain local estimates. To demonstrate this we include the proof of (3.10). 
Proof of (3.10).

From Verfürth [27], let $\psi \in Y$ with $\|\psi\|_{Y}=1$, then

$$
\langle F(u), \psi\rangle=\left\langle D F\left(u_{0}\right)\left(u-u_{0}\right), \psi\right\rangle+\left\langle\int_{0}^{1}\left[D F\left(u_{0}+t\left(u-u_{0}\right)\right)-D F\left(u_{0}\right)\right]\left(u-u_{0}\right) d t, \psi\right\rangle,
$$

and thus

$$
\begin{aligned}
\|F(u)\|_{Y^{*}} \leq & \left\|D F\left(u_{0}\right)\right\|_{\mathcal{L}\left(X, Y^{*}\right)}\left\|u-u_{0}\right\|_{X} \\
& +\int_{0}^{1}\left\|D F\left(u_{0}+t\left(u-u_{0}\right)\right)-D F\left(u_{0}\right)\right\|_{\mathcal{L}\left(X, Y^{*}\right)}\left\|u-u_{0}\right\|_{X} d t \\
\leq & \left\|D F\left(u_{0}\right)\right\|_{\mathcal{L}\left(X, Y^{*}\right)}\left\|u-u_{0}\right\|_{X}+\frac{1}{2} \Upsilon\left\|u-u_{0}\right\|_{X}^{2} \\
\leq & 2\left\|D F\left(u_{0}\right)\right\|_{\mathcal{L}\left(X, Y^{*}\right)}\left\|u-u_{0}\right\|_{X} .
\end{aligned}
$$

Now, let $S=\operatorname{span}\left\{\psi_{i}\right\} \subset Y$, where $\operatorname{support}\left(\psi_{i}\right) \subset \Lambda \subset \Omega$. Consider $\psi \in S$. In view of the proof of (3.10), we have that

$$
\begin{aligned}
\|F(u)\|_{S^{*}} & \leq 2\left\|D F\left(u_{0}\right)\right\|_{\mathcal{L}\left(X, S^{*}\right)}\left\|\left.\left(u-u_{0}\right)\right|_{\Lambda}\right\|_{X}, \\
& \leq 2\left\|D F\left(u_{0}\right)\right\|_{\mathcal{L}\left(X, Y^{*}\right)}\left\|\left.\left(u-u_{0}\right)\right|_{\Lambda}\right\|_{X} .
\end{aligned}
$$

Let $F_{h} \in C\left(X_{h}, Y_{h}^{*}\right)$ be an approximation of the function $F$. Denote the identity operator from $Y$ to $Y$ as $\operatorname{Id}_{Y}$. Then, we have the following result.

Theorem 3.2 (Verfürth [27]) Let $u_{h} \in X_{h}$ be an approximate solution of the equation

$$
F_{h}\left(u_{h}\right)=0,
$$

with $\left\|F_{h}\left(u_{h}\right)\right\|_{Y_{h}^{*}}$ "small". Assume that there is a restriction operator $\mathcal{R}_{h} \in \mathcal{L}\left(Y, Y_{h}\right)$, a finite dimensional subspace $\widetilde{Y}_{h}=\operatorname{span}\left\{\psi_{i}\right\} \subset Y$, where support $\left(\psi_{i}\right) \subset \Lambda \subset \Omega$, and an approximation $\widetilde{F}_{h}: X_{h} \rightarrow \widetilde{Y}_{h}^{*}$ of $F$ at $u_{h}$.

Then,

$$
\begin{aligned}
\left\|F\left(u_{h}\right)\right\|_{Y^{*}} \leq & \left\|\left(I d_{Y}-\mathcal{R}_{h}\right)^{*} \widetilde{F}_{h}\left(u_{h}\right)\right\|_{Y^{*}} \\
& +\left\|\left(I d_{Y}-\mathcal{R}_{h}\right)^{*}\left[F\left(u_{h}\right)-\widetilde{F}_{h}\left(u_{h}\right)\right]\right\|_{Y^{*}} \\
& +\left\|\mathcal{R}_{h}\right\|_{\mathcal{L}\left(Y, Y_{h}\right)}\left\|F\left(u_{h}\right)-F_{h}\left(u_{h}\right)\right\|_{Y_{h}^{*}} \\
& +\left\|\mathcal{R}_{h}\right\|_{\mathcal{L}\left(Y, Y_{h}\right)}\left\|F_{h}\left(u_{h}\right)\right\|_{Y_{h}^{*}}
\end{aligned}
$$

and

$$
\left\|\widetilde{F}_{h}\left(u_{h}\right)\right\|_{\widetilde{Y}_{h}^{*}} \leq\left\|F\left(u_{h}\right)\right\|_{\widetilde{Y}_{h}^{*}}+\left\|F\left(u_{h}\right)-\widetilde{F}_{h}\left(u_{h}\right)\right\|_{\widetilde{Y}_{h}^{*}}
$$

Combining Theorems 3.1 and 3.2 yields the basis for obtaining a residual based a posteriori error estimate. The choice of $\mathcal{R}_{h}$ comes naturally, given that $X_{h}$ and $Y_{h}$ are FE spaces(for example a Clément [6] type interpolation operator). Note that $\widetilde{F}_{h}\left(u_{h}\right)$ is a projection of $F\left(u_{h}\right)$ elementwise onto a suitable finite dimensional space. The space $\widetilde{Y}_{h}$ is typically the space spanned by a set of bubble functions constructed such that (3.16) holds. 


\subsection{FE approximation tools}

In this section we present a collection of FE tools used in constructing the error bounds. Let $W^{l, q}(\Omega)$ represent the usual Sobolev spaces with norm and seminorm $\|\cdot\|_{l, q ; \Omega}$ and $|\cdot|_{l, q ; \Omega}$, respectively. Likewise, the $L^{p}(\Omega)$ norm is defined as $\|\cdot\|_{p ; \Omega}$. In particular, let $H^{l}$ represent the Sobolev space $W^{l, 2}(\Omega)$ with norm and seminorm $\|\cdot\|_{l, 2 ; \Omega}$ and $|\cdot|_{l, 2 ; \Omega}$, respectively. For ease of notation we drop the domain from the norm and seminorm notations when the domain is obvious.

Let $\Pi_{h, j}(\Omega), j \geq 1$, denote a family of finite element partitions of $\Omega$ into simplices(triangles in $\mathbb{R}^{2}$ and tetrahedrons in $\mathbb{R}^{3}$ ) that satisfy:

1. Any two simplices in $\Pi_{h, j}(\Omega)$ are either disjoint or share a face, an edge or a vertex (a geometrically conforming partition).

2. The ratio $\frac{h_{T}}{\rho_{T}}<\varrho$, for all $T \in \Pi_{h, j}(\Omega)$, (no degenerate simplices),

where, $T, h_{T}, \rho_{T}$ and $h_{E}$ denote a simplex, the diameter of $T$, the diameter of the largest ball that can be inscribed into $T$, and the diameter of the face $E$ of $T$, respectively. These conditions allow local mesh refinements to be performed while preserving properties 1 and 2 .

Define $\mathcal{T}_{h}, \mathcal{E}_{h}$ and $\mathcal{N}_{h}$ as the collection of all the simplices, faces and vertices, respectively, in the partition $\Pi_{h, j}(\Omega)$. Given any $T \in \mathcal{T}_{h}$, denote by $\mathcal{E}_{h}(T)$ and $\mathcal{N}_{h}(T)$ the set of its faces and vertices respectively. For each $T \in \mathcal{T}_{h}$ and $E \in \mathcal{E}_{h}$, we define the neighborhoods and the extended neighborhoods of $T$ and $E$ respectively as:

$$
\begin{aligned}
& w_{T}:=\bigcup_{\mathcal{E}_{h}(T) \cap \mathcal{E}_{h}\left(T^{\prime}\right) \neq \emptyset} T^{\prime}, \quad w_{E}:=\bigcup_{E \in \mathcal{E}_{h}\left(T^{\prime}\right)} T^{\prime}, \\
& \widetilde{w}_{T}:=\bigcup_{\mathcal{N}_{h}(T) \cap \mathcal{N}\left(T^{\prime}\right) \neq \emptyset} T^{\prime}, \quad \widetilde{w}_{E}:=\bigcup_{\mathcal{N}_{h}(E) \cap \mathcal{N}\left(T^{\prime}\right) \neq \emptyset} T^{\prime},
\end{aligned}
$$

Let $W^{l, q}\left(\widetilde{w}_{T}\right)$ and $W^{l, q}\left(\widetilde{w}_{E}\right)$ be suitable Sobolev spaces defined on the extended neighborhoods of $T$ and $E$ respectively. For $k \in \mathbb{N}$, define

$$
\begin{aligned}
S_{h}^{k,-1} & :=\left\{\varphi: \Omega \rightarrow \mathbb{R}:\left.\varphi\right|_{T} \in \mathbb{P}_{k} \quad \forall T \in \Pi_{h, j}(\Omega)\right\}, \\
S_{h}^{k, 0} & :=S_{h}^{k,-1} \cap C(\bar{\Omega}),
\end{aligned}
$$

where, $\mathbb{P}_{k}, k \geq 0$, is the space of polynomials of degree at most $k$.

Let $\mathcal{I}_{h}: L^{1}(\Omega) \rightarrow S_{h}^{1,0}$ denote the interpolation operator of Clément [6]. Then, the following lemma holds.

Lemma 3.1 (Verfürth [27]) There exists constants $c_{1}$ and $c_{2}$ depending only on the ratio $\frac{h_{T}}{\rho_{T}}$ such that for $T \in \mathcal{T}_{h}, E \in \mathcal{E}_{h}$, and $1 \leq q \leq \infty$ the following error estimates are valid

$$
\begin{gathered}
\left\|\varphi-\mathcal{I}_{h} \varphi\right\|_{k, q ; T} \leq c_{1} h_{T}^{l-k}\|\varphi\|_{l, q ; \widetilde{w}_{T}} \quad, \quad 0 \leq k \leq l \leq 2, \quad \varphi \in W^{l, q}\left(\widetilde{w}_{T}\right), \\
\left\|\varphi-\mathcal{I}_{h} \varphi\right\|_{q ; E} \leq c_{2} h_{E}^{l-1 / q}\|\varphi\|_{l, q ; \widetilde{w}_{E}} \quad, \quad 1 \leq l \leq 2, \quad \varphi \in W^{l, q}\left(\widetilde{w}_{E}\right) .
\end{gathered}
$$


Given a partition of $\Omega$, we define the "broken" norms and seminorms on $\Omega$ as

$$
\|\cdot\|_{l, q ; \Omega}:=\left\{\sum_{T}\|\cdot\|_{l, q ; T}^{q}\right\}^{1 / q},\|\cdot\|_{q ; \Omega}:=\left\{\sum_{T}\|\cdot\|_{q ; T}^{q}\right\}^{1 / q},|\cdot|_{l, q ; \Omega}:=\left\{\sum_{T}|\cdot|_{l, q ; T}^{q}\right\}^{1 / q}
$$

Let $V_{T} \subset L^{\infty}(T)$ and $V_{E} \subset L^{\infty}(E)$ denote fixed polynomial spaces defined on $T$ and $E$ respectively. Let $\widehat{T}$ and $\widehat{E}$ be the reference simplex and reference face respectively as defined in [27]. Let $\psi_{\widehat{T}}$ and $\psi_{\widehat{E}} \in C^{\infty}(\widehat{T}, \mathbb{R})$ be two functions with the properties:

$$
\begin{array}{lll}
0 \leq \psi_{\widehat{T}} \leq 1, \quad \max _{\widehat{x} \in \widehat{T}} \psi_{\widehat{T}}(\widehat{x})=1, & \psi_{\widehat{T}}=0 & \text { on } \partial \widehat{T} \\
0 \leq \psi_{\widehat{E}} \leq 1, \quad \max _{\widehat{x} \in \widehat{E}} \psi_{\widehat{E}}(\widehat{x})=1, \quad \psi_{\widehat{E}}=0 & \text { on } \partial \widehat{T} \backslash \widehat{E} .
\end{array}
$$

Let $T$ be an arbitrary simplex and $E \subset \partial T$, then there is an invertible affine mapping

$$
F_{T}:=\widehat{T} \rightarrow T
$$

such that $\widehat{T}$ is mapped to $T$, and $\widehat{E}$ is mapped to $E$, and

$$
\psi_{T}:=\psi_{\widehat{T}} \circ F_{T}^{-1}, \quad \psi_{E}:=\psi_{\widehat{E}} \circ F_{T}^{-1} .
$$

For a simplex $T$ with face $E$, let $P: L^{\infty}(E) \rightarrow L^{\infty}(T)$ be the continuation operator defined in [27], and let $p$ and $q$ be two real numbers such that $\frac{1}{p}+\frac{1}{q}=1$, then the following lemma holds.

Lemma 3.2 (Verfürth [27]) There are constants $c_{1}, \ldots, c_{7}$ which only depend on the spaces $V_{\widehat{T}}$ and $V_{\widehat{E}}$, the functions $\psi_{\widehat{T}}$ and $\psi_{\widehat{E}}$, the number $p$ and the ratio $h_{T} / \rho_{T}$, such that the following inequalities hold for all $T \in \mathcal{T}_{h}, E \in \mathcal{E}_{h}, u \in V_{T}$, and $\sigma \in V_{E}$ :

$$
\begin{aligned}
& c_{1}\|u\|_{0, p ; T} \leq \sup _{v \in V_{T}} \frac{\int_{T} u \psi_{T} v}{\|v\|_{0, q ; T}} \leq\|u\|_{0, p ; T}, \\
& c_{2}\|\sigma\|_{p ; E} \leq \sup _{\tau \in V_{E}} \frac{\int_{E} \sigma \psi_{E} \tau}{\|\tau\|_{q ; E}} \leq\|\sigma\|_{p ; E}, \\
& c_{3} h_{T}^{-1}\left\|\psi_{T} u\right\|_{0, q ; T} \leq\left\|\nabla\left(\psi_{T} u\right)\right\|_{0, q ; T} \leq c_{4} h_{T}^{-1}\left\|\psi_{T} u\right\|_{0, q ; T}, \\
& c_{5} h_{T}^{-1}\left\|\psi_{E} P \sigma\right\|_{0, q ; T} \leq\left\|\nabla\left(\psi_{E} P \sigma\right)\right\|_{0, q ; T} \leq c_{6} h_{T}^{-1}\left\|\psi_{E} P \sigma\right\|_{0, q ; T}, \\
&\left\|\psi_{E} P \sigma\right\|_{0, q ; T} \leq c_{7} h_{T}^{1 / q}\|\sigma\|_{q ; E} .
\end{aligned}
$$

Similarly, by the definition of $\psi_{T}$,

$$
\psi_{T} u \leq u
$$

implying that

$$
\left\|\psi_{T} u\right\|_{0, p ; T} \leq\|u\|_{0, p ; T}
$$

In addition we have the following local inverse inequalities. 
Lemma 3.3 ([15]) There exists a positive constant $C$, depending on the shape regularity constant, such that for all $u \in \mathbb{P}_{k}(T)$ and for all $T \in \Pi_{h, j}(\Omega)$

$$
\|u\|_{2 ; \partial T}^{2} \leq C \frac{k^{2}}{h_{T}}\|u\|_{2 ; T}^{2}, \quad|u|_{1,2 ; T}^{2} \leq C \frac{k^{4}}{h_{T}^{2}}\|u\|_{2 ; T}^{2}
$$

\section{A Posteriori Error Estimation for Viscoelastic Fluid Flow}

Using the theorems and lemmas above, combined with standard tools used in FE methods, one can now proceed with the construction of a posteriori error estimates for viscoelastic fluid flow governed by differential constitutive laws. Before we proceed let us define the spaces to be used.

$$
\begin{array}{rll}
\text { Velocity Space: } & M:=H_{0}^{1}(\Omega)^{n}=\left\{v \in H^{1}(\Omega)^{n}: v=0 \text { on } \partial \Omega\right\}, \\
\text { Stress Space: } & \Sigma:=H^{1}(\Omega)_{\text {sym }}^{n \times n}=\left\{\sigma_{i j} \in H^{1}(\Omega), 1 \leq i, j \leq n: \quad \sigma_{i j}=\sigma_{j i}\right\} \\
\text { Pressure Space: } & Q:=L_{0}^{2}(\Omega)=\left\{q \in L^{2}(\Omega): \int_{\Omega} q d x=0\right\} .
\end{array}
$$

Given these spaces we can then define the variational form for problem (2.4)-(2.7) as:

$$
\begin{aligned}
\langle F([u, \tau, p]),[v, \sigma, q]\rangle:= & \\
\int_{\Omega}(\tau & \left.+\lambda(u \cdot \nabla) \tau+\lambda \frac{\gamma}{\alpha}(\tau \tau)+\lambda g_{a}(\tau, \nabla u)-2 \alpha \mathbf{D}(u)\right): \sigma d A \\
& +\int_{\Omega}((2(1-\alpha) \mathbf{D}(u)+\tau-p \mathbb{I}): \nabla v-f \cdot v) d A \\
& +\int_{\Omega} q \nabla \cdot u d A, \quad \forall[v, \sigma, q] \in[M, \Sigma, Q] .
\end{aligned}
$$

Let $M_{h} \subset M, \Sigma_{h} \subset \Sigma$ and $Q_{h} \subset Q$ be the FE spaces corresponding to a partition, $\Pi_{h, j}(\Omega)$ of $\Omega$. Define $M_{h}, \Sigma_{h}$ and $Q_{h}$ as:

$$
\begin{aligned}
M_{h} & :=\left\{v \in M \cap \mathbf{C}^{0}(\bar{\Omega})^{n} ; v \in \mathbb{P}_{k}(T)^{n}, \forall T \in \Pi_{h, j}(\Omega)\right\}, \\
\Sigma_{h} & :=\left\{\sigma \in \Sigma \cap \mathbf{C}^{0}(\bar{\Omega})^{n \times n} ; \sigma \in \mathbb{P}_{l}(T)^{n \times n}, \forall T \in \Pi_{h, j}(\Omega)\right\}, \\
Q_{h} & :=\left\{q \in Q \cap \mathbf{C}^{0}(\bar{\Omega}) ; q \in \mathbb{P}_{s}(T), \forall T \in \Pi_{h, j}(\Omega)\right\},
\end{aligned}
$$

where $k$ and $s$ are properly chosen so as to satisfy the LBB (inf-sup) condition [12]. If $M_{h}$ and $Q_{h}$ do not satisfy the inf-sup condition, then one needs to adopt an appropriate stabilization mechanism for the modified Stokes part of the governing equations (see [21] for some stabilization techniques).

A discretization of $F$ using SUPG as the stabilization mechanism for the constitutive equation then 
yields $F_{h}$ as:

$$
\begin{aligned}
& \left\langle F_{h}\left(\left[u_{h}, \tau_{h}, p_{h}\right]\right),\left[v_{h}, \sigma_{h}, q_{h}\right]\right\rangle:=\left\langle F\left(\left[u_{h}, \tau_{h}, p_{h}\right]\right),\left[v_{h}, \sigma_{h}, q_{h}\right]\right\rangle \\
& +\sum_{T} \int_{T}\left(\tau_{h}+\lambda\left(u_{h} \cdot \nabla\right) \tau_{h}+\lambda \frac{\gamma}{\alpha}\left(\tau_{h} \tau_{h}\right)+\lambda g_{a}\left(\tau_{h}, \nabla u_{h}\right)-2 \alpha \mathbf{D}\left(u_{h}\right)\right):\left(\delta\left(h_{T}, u_{h}\right) u_{h} \cdot \nabla \sigma_{h}\right) d A, \\
& \forall\left[v_{h}, \sigma_{h}, q_{h}\right] \in\left[M_{h}, \Sigma_{h}, Q_{h}\right], \quad(4.35)
\end{aligned}
$$

where

$$
\delta\left(h_{T}, u_{h}\right)=\left\{\begin{array}{cl}
\frac{c h_{T}|T|^{1 / 2}}{\lambda\left\|u_{h}\right\|_{0,2 ; T}}, & \text { if } u_{h} \neq 0 \text { on } T \\
0, & \text { if } u_{h}=0 \text { on } T
\end{array},\right.
$$

with $|T|$ denoting the area/volume of $T$ in $\mathbb{R}^{n}$.

In [23], Sandri proved solvability of (4.35) for the Oldroyd-B model $(\gamma=0)$ for $k=2, l=1, s=1$, and established a priori error estimates for the approximation, assuming a smooth, small solution.

Let $\pi_{k, T} y$ be a projection of $y$ onto a polynomial space with degree $k$ on the simplex $T$. Next, we define $\widetilde{F}_{h}$ as $F$ in (4.31), with $f$ replaced by its projection $\pi_{k_{u}, T} f$, where $k_{u}$ is the polynomial degree of the velocity space. Then, the following equality is straight forward to verify.

$$
\begin{aligned}
& \left\langle\widetilde{F}_{h}\left(\left[u_{h}, \tau_{h}, p_{h}\right]\right),[v, \sigma, q]\right\rangle= \\
& \sum_{T} \int_{T}\left(\tau_{h}+\lambda\left(u_{h} \cdot \nabla\right) \tau_{h}+\lambda \frac{\gamma}{\alpha}\left(\tau_{h} \tau_{h}\right)+\lambda g_{a}\left(\tau_{h}, \nabla u_{h}\right)-2 \alpha \mathbf{D}\left(u_{h}\right)\right): \sigma d A \\
& \quad+\sum_{T} \int_{T}\left(-2(1-\alpha) \nabla \cdot \mathbf{D}\left(u_{h}\right)-\nabla \cdot \tau_{h}+\nabla p_{h}-\pi_{k_{u}, T} f\right) \cdot v d A \\
& +\sum_{T} \int_{T} q \nabla \cdot u_{h} d A+\sum_{E} \int_{E}\left[\tau_{h} \cdot \mathbf{n}_{E}-p_{E}+2(1-\alpha) \mathbf{D}\left(u_{h}\right) \cdot \mathbf{n}_{E}\right]_{E} \cdot v d S, \\
& \forall[v, \sigma, q] \in[M, \Sigma, Q],
\end{aligned}
$$

where the notation $[f]_{E}$ represents the jump of $f$ across the face $E$ and $\mathbf{n}_{E}$ is the normal vector to $E$. If $E$ falls on $\Gamma$ then $\mathbf{n}_{E}$ coincides with the exterior normal on $\Gamma$.

Note that the above definitions of $F, F_{h}$, and $\widetilde{F}_{h}$ depend on the formulation adopted, and the stabilization mechanism used.

For ease of notation define $X=Y:=M \times \Sigma \times Q$ and $X_{h}=Y_{h}:=M_{h} \times \Sigma_{h} \times Q_{h}$. For $x=(u, \tau, p) \in Y$ define the norm on $Y$ as

$$
\|x\|_{X}=\|x\|_{Y}=\left\{\|u\|_{1,2}^{2}+\|\tau\|_{1,2}^{2}+\|p\|_{0,2}^{2}\right\}^{1 / 2} .
$$

Also, for brevity let

$$
\begin{aligned}
R_{m} & :=-\nabla \cdot \tau_{h}-2(1-\alpha) \nabla \cdot \mathbf{D}\left(u_{h}\right)+\nabla p_{h}-\pi_{k_{u}, T} f \\
R_{s} & :=\tau_{h}+\lambda\left(u_{h} \cdot \nabla\right) \tau_{h}+\lambda \frac{\gamma}{\alpha}\left(\tau_{h} \tau_{h}\right)+\lambda g_{a}\left(\tau_{h}, \nabla u_{h}\right)-2 \alpha \mathbf{D}\left(u_{h}\right), \\
R_{c} & :=\nabla \cdot u_{h} \\
R_{J} & :=\left[\tau_{h} \cdot \mathbf{n}_{E}-p_{h} \mathbf{n}_{E}+2(1-\alpha) \mathbf{D}\left(u_{h}\right) \cdot \mathbf{n}_{E}\right]_{E} .
\end{aligned}
$$


Before we proceed let us define the restriction operator $\mathcal{R}_{h}: Y \rightarrow Y_{h}$ as

$$
\mathcal{R}_{h}:=\left[\mathcal{I}_{h} u_{1}, \ldots, \mathcal{I}_{h} u_{n}, \mathcal{I}_{h} \tau_{11}, \mathcal{I}_{h} \tau_{12}, \ldots, \mathcal{I}_{h} \tau_{n n}, 0\right]
$$

Let the polynomial degrees of the approximating spaces for $u, \tau$ and $p$ be $k, l$ and $s$ respectively. Then, the space $\widetilde{Y}_{h}$ is defined as

$$
\begin{aligned}
& \tilde{Y}_{h}:=\operatorname{span}\left\{\left[\psi_{T} v, \underline{\underline{0}}, 0\right],\left[\underline{0}, \psi_{T} \sigma, 0\right],\left[\underline{0}, \underline{\underline{0}}, \psi_{T} q\right],\left[\psi_{E} P w, \underline{\underline{0}}, 0\right]:\right. \\
& v\left.\in\left[\mathbb{P}_{m_{1}}(T)\right]^{n}, \sigma \in\left[\mathbb{P}_{m_{2}}(T)\right]^{n \times n}, q \in\left[\mathbb{P}_{k-1}(T)\right], w \in\left[\mathbb{P}_{m_{3}}(E)\right]^{n},\right\}
\end{aligned}
$$

where $P$ is the continuation operator defined in [27], $\underline{0}$ is the zero vector, $\underline{\underline{0}}$ is the zero tensor and

$$
\begin{aligned}
& m_{1}=\max \{k, l-1, s-1\}, \\
& m_{2}=\max \{k+l-1, k-1,2 l\}, \\
& m_{3}=\max \{k-1, l, s\} .
\end{aligned}
$$

Given the spaces and auxillary results above, we now construct the necessary bounds to the terms in (3.15) and (3.16). First, we construct an upper bound for the term $\left\|\left(\operatorname{Id}_{Y}-\mathcal{R}_{h}\right)^{*} \widetilde{F}_{h}\left(\left[u_{h}, \tau_{h}, p_{h}\right]\right)\right\|_{Y^{*}}$. Let $[v, \sigma, q] \in Y$ with $\|[v, \sigma, q]\|_{Y}=1$, then using Lemma 3.1,

$$
\begin{aligned}
\left\|\left(\operatorname{Id}_{Y}-\mathcal{R}_{h}\right)^{*} \widetilde{F}_{h}\left(\left[u_{h}, \tau_{h}, p_{h}\right]\right)\right\|_{Y^{*}} & =\sup _{\|[v, \sigma, q] \in Y} \mid \sum_{T} \int_{T} R_{s}:\left(\sigma-\mathcal{I}_{h} \sigma\right) d A+\sum_{T} \int_{T} R_{m} \cdot\left(v-\mathcal{I}_{h} v\right) d A \\
& +\sum_{T} \int_{T} q R_{c} d A+\sum_{E} \int_{E} R_{J} \cdot\left(v-\mathcal{I}_{h} v\right) d S \mid \\
& \leq c\left\{\sum_{T} \eta_{T}^{2}\right\}^{1 / 2},
\end{aligned}
$$

where $\eta_{T}$ is defined as

$$
\begin{aligned}
\eta_{T}:= & \left\{h_{T}^{2}\left\|\tau_{h}+\lambda\left(u_{h} \cdot \nabla\right) \tau_{h}+\lambda g_{a}\left(\tau_{h}, \nabla u_{h}\right)+\lambda \frac{\gamma}{\alpha}\left(\tau_{h} \tau_{h}\right)-2 \alpha \mathbf{D}\left(u_{h}\right)\right\|_{0,2 ; T}^{2}\right. \\
& +h_{T}^{2}\left\|-\nabla \cdot \tau_{h}-2(1-\alpha) \nabla \cdot \mathbf{D}\left(u_{h}\right)+\nabla p_{h}-\pi_{k_{u}, T} f\right\|_{0,2 ; T}^{2} \\
& \left.+\left\|\nabla \cdot u_{h}\right\|_{0,2 ; T}^{2}+h_{E}\left\|\left[\tau_{h} \cdot \mathbf{n}_{E}-p_{h} \mathbf{n}_{E}+2(1-\alpha) \mathbf{D}\left(u_{h}\right) \cdot \mathbf{n}_{E}\right]_{E}\right\|_{2 ; E}^{2}\right\}^{1 / 2} .
\end{aligned}
$$

Next, using Lemma 3.1 we can bound the second term in (3.15) as:

$$
\begin{aligned}
\left\|\left(\operatorname{Id}_{Y}-\mathcal{R}_{h}\right)^{*}\left[F\left(\left[u_{h}, \tau_{h}, p_{h}\right]\right)-\widetilde{F}_{h}\left(\left[u_{h}, \tau_{h}, p_{h}\right]\right)\right]\right\| \|_{Y^{*}} \\
=\sup _{\substack{[v, \sigma, q] \in Y \\
\| v, \sigma, q] \|_{Y=1}}}\left|\sum_{T} \sum_{i=1}^{n} \int_{T}\left(f_{i}-\pi_{k_{u}, T} f_{i}\right)\left(v_{i}-\mathcal{I}_{h} v_{i}\right) d A\right| \\
\leq c \sum_{T}\left\|f-\pi_{k_{u}, T} f\right\|_{0,2 ; T}\left\|v-\mathcal{I}_{h} v\right\|_{0,2 ; T} \\
\leq c\left\{\sum_{T} h_{T}^{2}\left\|f-\pi_{k_{u}, T} f\right\|_{0,2 ; T}^{2}\right\}^{1 / 2}
\end{aligned}
$$


Now, let us examine the consistency error. Let $\left[v_{h}, \sigma_{h}, q_{h}\right] \in Y_{h}$ then,

$$
\left\langle F\left(\left[u_{h}, \tau_{h}, p_{h}\right]\right)-F_{h}\left(\left[u_{h}, \tau_{h}, p_{h}\right]\right),\left[v_{h}, \sigma_{h}, q_{h}\right]\right\rangle=-\sum_{T} \delta\left(h_{T}, u_{h}\right) \int_{T} R_{s}:\left(u_{h} \cdot \nabla \sigma_{h}\right) d A .
$$

By definition,

$$
\begin{aligned}
\| F\left(\left[u_{h}, \tau_{h}, p_{h}\right]\right)- & F_{h}\left(\left[u_{h}, \tau_{h}, p_{h}\right]\right) \|_{Y_{h}^{*}} \\
= & \sup _{\substack{\left[v_{h}, \sigma_{h}, q_{h}\right] \in Y_{h} \\
\left\|\left[v_{h}, \sigma_{h}, q_{h}\right]\right\|_{Y}=1}}\left|\left\langle F\left(\left[u_{h}, \tau_{h}, p_{h}\right]\right)-F_{h}\left(\left[u_{h}, \tau_{h}, p_{h}\right]\right),\left[v_{h}, \sigma_{h}, q_{h}\right]\right\rangle\right| \\
= & \sup _{\substack{\left[v_{h}, \sigma_{h}, q_{h}\right] \in Y_{h}\\
}}\left|\sum_{T} \delta\left(h_{T}, u_{h}\right) \int_{T} R_{s}:\left(u_{h} \cdot \nabla \sigma_{h}\right) d A\right| \\
\leq & \sum_{T} \delta\left(h_{T}, u_{h}\right)\left\|R_{s}\right\|_{0,2 ; T}\left\|u_{h} \cdot \nabla \sigma_{h}\right\|_{0,2 ; T} \\
\leq & c \sum_{T} \delta\left(h_{T}, u_{h}\right)\left\|R_{s}\right\|_{0,2 ; T}\left\|u_{h}\right\|_{\infty, T}\left\|\nabla \sigma_{h}\right\|_{0,2 ; T} \\
\leq & c \sum_{T} \delta\left(h_{T}, u_{h}\right)\left\|R_{s}\right\|_{0,2 ; T}\left\|u_{h}\right\|_{\infty, T}\left\|\sigma_{h}\right\|_{1,2 ; T} .
\end{aligned}
$$

Thus,

$$
\left\|F\left(\left[u_{h}, \tau_{h}, p_{h}\right]\right)-F_{h}\left(\left[u_{h}, \tau_{h}, p_{h}\right]\right)\right\|_{Y_{h}^{*}} \leq c\left\{\sum_{T}\left(\left\|u_{h}\right\|_{\infty, T} \delta\left(h_{T}, u_{h}\right)\right)^{2}\left\|R_{s}\right\|_{0,2 ; T}^{2}\right\}^{1 / 2} .
$$

The term $\left\|F_{h}\left(u_{h}\right)\right\|_{Y_{h}^{*}}$ in Proposition 3.2 represents the residual of the approximating linear system. Since we assume the system is solved "exactly" (up to round-off error) we have

$$
\left\|F_{h}\left(u_{h}\right)\right\|_{Y_{h}^{*}}=0
$$

Equations (3.16) and (3.14) can be combined to obtain a local lower bound for the error estimate. This is done in two steps. First, we show that we can bound each of the terms in $\eta_{T}$ by $\sup \left\langle\widetilde{F}_{h}\left(\left[u_{h}, \tau_{h}, p_{h}\right]\right),\left[v_{b}, \sigma_{b}, q_{b}\right]\right\rangle$ for $\left[v_{b}, \sigma_{b}, q_{b}\right] \in \widetilde{Y}_{h}$ with $\operatorname{support}\left(\left[v_{b}, \sigma_{b}, q_{b}\right]\right) \subset w_{T}$ and $\left\|\left[v_{b}, \sigma_{b}, q_{b}\right]\right\|_{Y}=$ 1. Then, we construct an upper bound for the second term on the right hand side of (3.16).

Let $\widetilde{Y}_{h} \mid w_{0}, w_{0} \in\left\{T, w_{E}, w_{T}\right\}$, denote the set of all functions $\phi \in \widetilde{Y}_{h}$ with support in $w_{0}$. Using Lemma 3.2 we start with the residual term from the continuity equation.

$$
\begin{aligned}
c_{1}\left\|R_{c}\right\|_{0,2 ; T} & \leq \sup _{s \in \mathbb{P}_{k-1}(T) \backslash\{0\}} \frac{\int_{T}\left(\nabla \cdot u_{h}\right) \psi_{T} s d A}{\|s\|_{0,2 ; T}} \\
c_{1}\left\|R_{c}\right\|_{0,2 ; T} & \leq \sup _{s \in \mathbb{P}_{k-1}(T) \backslash\{0\}} \frac{\left\langle\widetilde{F}_{h}\left(\left[u_{h}, \tau_{h}, p_{h}\right]\right),\left[\underline{0}, \underline{\underline{0}}, \psi_{T} s\right]\right\rangle}{\left\|\psi_{T} s\right\|_{0,2 ; T}} \\
& \leq \sup _{\substack{\left.\left[v_{b}, \sigma_{b}, q_{b}\right] \in \widetilde{Y}_{h}\right|_{T} \\
\|\left[v_{b}, \sigma_{b}, q_{b}\right.} \|_{Y=1}}\left\langle\widetilde{F}_{h}\left(\left[u_{h}, \tau_{h}, p_{h}\right]\right),\left[v_{b}, \sigma_{b}, q_{b}\right]\right\rangle .
\end{aligned}
$$


Next, the residual from the momentum equation. By combining the lower and upper bound from (3.24) and (3.26) we have,

$$
\begin{aligned}
c_{1}\left\|R_{m}\right\|_{0,2 ; T} & \leq \sup _{w \in \mathbb{P}_{m_{1}}(T) \backslash\{0\}} \frac{\int_{T} R_{m} \cdot \psi_{T} w d A}{\|w\|_{0,2 ; T}} \\
c_{1} c_{4}^{-1} h_{T}\left\|R_{m}\right\|_{0,2 ; T} & \leq \sup _{w \in \mathbb{P}_{m_{1}}(T) \backslash\{0\}} \frac{\int_{T} R_{m} \cdot \psi_{T} w d A}{\left\|\nabla\left(\psi_{T} w\right)\right\|_{0,2 ; T}} \\
& =\sup _{w \in \mathbb{P}_{m_{1}}(T) \backslash\{0\}} \frac{\left\langle\widetilde{F}_{h}\left(\left[u_{h}, \tau_{h}, p_{h}\right]\right),\left[\psi_{T} w, \underline{\underline{0}}, 0\right]\right\rangle}{\left\|\nabla\left(\psi_{T} w\right)\right\|_{0,2 ; T}} \\
& \leq c \sup _{\substack{\left.\left[v_{b}, \sigma_{b}, q_{b}\right] \in \tilde{Y}_{h}\right|_{T} \\
\left\|\left[v_{b}, \sigma_{b}, q_{b}\right]\right\|_{Y}=1}}^{\left\langle\widetilde{F}_{h}\left(\left[u_{h}, \tau_{h}, p_{h}\right]\right),\left[v_{b}, \sigma_{b}, q_{b}\right]\right\rangle .}
\end{aligned}
$$

The constitutive equation term is then bounded in a similar fashion to the momentum equation

$$
\begin{aligned}
c_{1}\left\|R_{s}\right\|_{0,2 ; T} & \leq \sup _{r \in \mathbb{P}_{m_{2}}(T) \backslash\{0\}} \frac{\int_{T} R_{s}: \psi_{T} r d A}{\|r\|_{0,2 ; T}} \\
c_{1}\left\|R_{s}\right\|_{0,2 ; T} & \leq \sup _{r \in \mathbb{P}_{m_{2}}(T) \backslash\{0\}} \frac{\left\langle\widetilde{F}_{h}\left(\left[u_{h}, \tau_{h}, p_{h}\right]\right),\left[\underline{0}, \psi_{T} r, 0\right]\right\rangle}{\left\|\psi_{T} r\right\|_{0,2 ; T}} \\
c_{1} c_{4}^{-1} h_{T}\left\|R_{s}\right\|_{0,2 ; T} & \leq \sup _{r \in \mathbb{P}_{m_{2}}(T) \backslash\{0\}} \frac{\left\langle\widetilde{F}_{h}\left(\left[u_{h}, \tau_{h}, p_{h}\right]\right),\left[\underline{0}, \psi_{T} r, 0\right]\right\rangle}{\left\|\nabla\left(\psi_{T} r\right)\right\|_{0,2 ; T}} \\
& \leq \sup _{\substack{\left.\left[v_{b}, \sigma_{b}, q_{b}\right] \in \tilde{Y}_{h}\right|_{T} \\
\left\|\left[v_{b}, \sigma_{b}, q_{b}\right]\right\|_{Y}=1}}\left\langle\widetilde{F}_{h}\left(\left[u_{h}, \tau_{h}, p_{h}\right]\right),\left[v_{b}, \sigma_{b}, q_{b}\right]\right\rangle .
\end{aligned}
$$

Lastly, the jump term. We begin by establishing that for $t \in \mathbb{P}_{m_{3}}(E)$ we have

$$
\left\|\left.t\right|_{2 ; E} \geq c h_{E}^{1 / 2}\right\| \psi_{E} P t \|_{1,2 ; w_{E}} .
$$

Letting $T_{i}, \quad i=1,2$ denote the simplices which share the face $E$, from the upper bounds in (3.28) and (3.27) it follows that

$$
\begin{aligned}
\|t\|_{2 ; E} & \geq c_{7}^{-1} h_{T_{i}}^{-1 / 2}\left\|\psi_{E} P t\right\|_{0,2 ; T_{i}}=\frac{1}{2} c_{7}^{-1} h_{T_{i}}^{-1 / 2}\left\|\psi_{E} P t\right\|_{0,2 ; T_{i}}+\frac{1}{2} c_{7}^{-1} h_{T_{i}}^{-1 / 2}\left\|\psi_{E} P t\right\|_{0,2 ; T_{i}} \\
& \geq \frac{1}{2} c_{7}^{-1} h_{T_{i}}^{-1 / 2}\left\|\psi_{E} P t\right\|_{0,2 ; T_{i}}+\frac{1}{2} c_{7}^{-1} h_{T_{i}}^{-1 / 2} c_{6}^{-1} h_{T_{i}}\left\|\nabla \psi_{E} P t\right\|_{0,2 ; T_{i}} \\
& \geq \frac{1}{\sqrt{2}} c h_{T_{i}}^{1 / 2}\left\|\psi_{E} P t\right\|_{1,2 ; T_{i}} .
\end{aligned}
$$

As $w_{E}=T_{1} \cup T_{2}$ and $\frac{h_{T}}{\rho_{T}}<\varrho$, estimate (4.55) follows. 
Now, from (3.25),(3.27) and (4.55),

$$
\begin{aligned}
c_{2}\left\|R_{J}\right\|_{2 ; E} & \leq \sup _{t \in \mathbb{P}_{m_{3}}(E) \backslash\{0\}} \frac{\int_{E} R_{J} \cdot \psi P t d A}{\|t\|_{2 ; E}} \\
& \leq \sup _{t \in \mathbb{P}_{m_{3}}(E) \backslash\{0\}} \frac{\left\{\left\langle\widetilde{F}_{h}\left(\left[u_{h}, \tau_{h}, p_{h}\right]\right),\left[\psi_{E} P t, \underline{\underline{0}}, 0\right]\right\rangle-\int_{w_{E}} R_{m} \cdot \psi_{E} P t d A\right\}}{c h_{E}^{1 / 2}\left\|\psi_{E} P t\right\|_{1,2 ; w_{E}}} \\
& \leq \sup _{t \in \mathbb{P}_{m_{3}}(E) \backslash\{0\}} \frac{\left\langle\widetilde{F}_{h}\left(\left[u_{h}, \tau_{h}, p_{h}\right]\right),\left[\psi_{E} P t, \underline{\underline{0}}, 0\right]\right\rangle+\left\|R_{m}\right\|_{0,2 ; w_{E}}\left\|\psi_{E} P t\right\|_{0,2 ; w_{E}}}{c h_{E}^{1 / 2}\left\|\psi_{E} P t\right\|_{1,2 ; w_{E}}} \\
& \leq \sup _{t \in \mathbb{P}_{m_{3}}(E) \backslash\{0\}} \frac{\left\langle\widetilde{F}_{h}\left(\left[u_{h}, \tau_{h}, p_{h}\right]\right),\left[\psi_{E} P t, \underline{\underline{0}}, 0\right]\right\rangle+c_{5} h_{T}\left\|R_{m}\right\|_{0,2 ; w_{E}}\left\|\nabla\left(\psi_{E} P t\right)\right\|_{0,2 ; w_{E}}}{c h_{E}^{1 / 2}\left\|\psi_{E} P t\right\|_{1,2 ; w_{E}}} .
\end{aligned}
$$

Combining (4.59) and (4.53) we obtain

$$
h_{E}^{1 / 2}\left\|R_{J}\right\|_{2 ; E} \leq \tilde{C} \sup _{\substack{\left.\left[v_{b}, \sigma_{b}, q_{b}\right] \in \widetilde{Y}_{h}\right|_{w_{E}} \\\left\|\left[v_{b}, \sigma_{b}, q_{b}\right]\right\|_{Y}=1}}\left\langle\widetilde{F}_{h}\left(\left[u_{h}, \tau_{h}, p_{h}\right]\right),\left[v_{b}, \sigma_{b}, q_{b}\right]\right\rangle .
$$

Now, combining inequalities $(4.52),(4.53),(4.54)$ and (4.60) we get

$$
\eta_{T} \leq c \sup _{\substack{\left.\left[v_{b}, \sigma_{b}, q_{b}\right] \in \tilde{Y}_{h}\right|_{w_{T}} \\\left\|\left[v_{b}, \sigma_{b}, q_{b}\right]\right\|_{Y}=1}}\left\langle\widetilde{F}_{h}\left(\left[u_{h}, \tau_{h}, p_{h}\right]\right),\left[v_{b}, \sigma_{b}, q_{b}\right]\right\rangle .
$$

Next, the second part of (3.16). Using Lemma 3.2 and $\left[v_{b}, \sigma_{b}, q_{b}\right] \in \widetilde{Y}_{h}$, we now construct an upper bound for the second term on the right hand side of (3.16).

As,

$$
\left\langle F\left(\left[u_{h}, \tau_{h}, p_{h}\right]\right)-\widetilde{F}_{h}\left(\left[u_{h}, \tau_{h}, p_{h}\right]\right),\left[v_{b}, \sigma_{b}, q_{b}\right]\right\rangle=\sum_{T^{\prime} \subset w_{T}} \int_{T^{\prime}}\left(f-\pi_{k_{u}, T^{\prime}} f\right) \cdot v_{b} d A
$$

we have

$$
\begin{aligned}
\left\|F\left(\left[u_{h}, \tau_{h}, p_{h}\right]\right)-\widetilde{F}_{h}\left(\left[u_{h}, \tau_{h}, p_{h}\right]\right)\right\|_{\widetilde{Y}_{h}^{*}} & =\sup _{\substack{\left[v_{b}, \sigma_{b}, q_{b} b \in \tilde{Y}_{h} \\
\left\|\left[v_{b}, \sigma_{b}, q_{b}\right]\right\|_{Y}=1\right.}} \frac{\left|\left\langle F\left(\left[u_{h}, \tau_{h}, p_{h}\right]\right)-\widetilde{F}_{h}\left(\left[u_{h}, \tau_{h}, p_{h}\right]\right),\left[v_{b}, \sigma_{b}, q_{b}\right]\right\rangle\right|}{\left\|\left[v_{b}, \sigma_{b}, q_{b}\right]\right\|_{Y}} \\
& \leq \sup _{\substack{\left[v_{b}, \sigma_{b}, q_{b}\right] \in \tilde{Y}_{h} \\
\left\|\left[v_{b}, \sigma_{b}, q_{b}\right]\right\|_{Y}=1}} \sum_{T}\left|\int_{T}\left(f-\pi_{k_{u}, T} f\right) \cdot v_{b} d A\right| \\
& \leq \sum_{T^{\prime} \subset w_{T}}\left\|f-\pi_{k_{u}, T^{\prime}} f\right\|_{0,2 ; T^{\prime}}\left\|v_{b}\right\|_{0,2 ; T^{\prime}} \\
& \leq c_{3}^{-1} \sum_{T^{\prime} \subset w_{T}} h_{T^{\prime}}\left\|f-\pi_{k_{u}, T^{\prime}} f\right\|_{0,2 ; T^{\prime}}\left\|\nabla v_{b}\right\|_{0,2 ; T^{\prime}} \\
& \leq c_{3}^{-1}\left\{\sum_{T^{\prime} \subset w_{T}} h_{T^{\prime}}^{2}\left\|f-\pi_{k_{u}, T^{\prime}} f\right\|_{0,2 ; T^{\prime}}^{2}\right\}^{1 / 2} .
\end{aligned}
$$


Combining (4.61) and (4.63) with (3.14) and (3.16) yields

$$
\begin{aligned}
\eta_{T} \leq & \bar{c}_{4}\left\{\left\|u-u_{h}\right\|_{1,2, w_{T}}^{2}+\left\|\tau-\tau_{h}\right\|_{1,2, w_{T}}^{2}+\left\|p-p_{h}\right\|_{0,2, w_{T}}^{2}\right\}^{1 / 2} \\
& +\bar{c}_{5}\left\{\sum_{T^{\prime} \subset w_{T}} h_{T^{\prime}}^{2}\left\|f-\pi_{k_{u}, T^{\prime}} f\right\|_{0,2 ; T^{\prime}}^{2}\right\}^{1 / 2} .
\end{aligned}
$$

Next, we combine the above computations with Theorem 3.1 to establish an a posteriori error estimate. However, before doing so we comment on the subspaces $X_{D} \subset X$ and $Y_{D}^{*} \subset Y^{*}$. For $F(u)=0$ denoting the system of equations (2.4)-(2.6), and $X=Y=M \times \Sigma \times Q, D F\left(u_{0}\right) \notin$ $\operatorname{Isom}\left(X, Y^{*}\right)$. From [10], we have that for $\Omega \subset \mathbb{R}^{n}(n=2,3), \partial \Omega \in C^{2}, \gamma=0$, if $f \in L^{r}(n<r<\infty)$ has a sufficiently small $L^{r}$-norm then (2.4)-(2.6) possesses exactly one small strong solution $(u, \tau, p)$,

$$
u \in D\left(A_{r}\right), \quad \tau \in W^{1, r}(\Omega)_{s y m}^{n \times n}, \quad p \in \widetilde{W}^{1, r}(\Omega),
$$

where $W^{p, q}$ denotes the usual Sobolev space notation, and

$$
\begin{gathered}
D\left(A_{r}\right):=W^{2, r}(\Omega)^{n} \cap W_{0}^{1, r}(\Omega)^{n} \cap\left\{v \in L^{r}(\Omega)^{n}: \nabla \cdot v=0, v \cdot \mathbf{n}=0 \text { on } \partial \Omega\right\}, \\
\widetilde{W}^{1, r}(\Omega)=\left\{v \in W^{1, r}(\Omega) \mid \int_{\Omega} v d x=0\right\} .
\end{gathered}
$$

For

$$
\widehat{X}_{D}=\left(W^{2, r}(\Omega)^{n} \cap W_{0}^{1, r}(\Omega)^{n}\right) \times W^{1, r}(\Omega)_{s y m}^{n \times n} \times \widetilde{W}^{1, r}(\Omega),
$$

and

$$
\widehat{Y}_{D}^{*}=L^{r}(\Omega)^{n} \times W^{1, r}(\Omega)_{s y m}^{n \times n} \times W^{1, r}(\Omega),
$$

the iterative argument used in [10] to establish existence and uniqueness of the strong solution may be modified to show that for $u_{0} \in\left(W^{2, r}(\Omega)^{n} \cap W_{0}^{1, r}(\Omega)^{n}\right) \times W^{2, r}(\Omega)_{\text {sym }}^{n \times n} \times \widetilde{W}^{1, r}(\Omega)$ sufficiently small, $D F\left(u_{0}\right) \in \operatorname{Isom}\left(\widehat{X}_{D}, \widehat{Y}_{D}^{*}\right)$.

To obtain an a posteriori error estimate we let $u$ in (3.11) denote the approximation $\left(u_{h}, \tau_{h}, p_{h}\right)$. Additionally, we must assume the existence of subspaces $X_{D} \subset X$ and $Y_{D}^{*} \subset Y^{*}$ such that the weak solution $(u, \tau, p) \in X_{D},\left(u_{h}, \tau_{h}, p_{h}\right) \in X_{D}$, and $D F(u, \tau, p) \in \operatorname{Isom}\left(X_{D}, Y_{D}^{*}\right)$. (Note that we cannot use the strong solution space $\widehat{X}_{D}$ for our choice of $X_{D}$ as $u_{h} \notin W^{2, r}(\Omega)$.)

Theorem 4.1 Let $\left[u_{0}, \tau_{0}, p_{0}\right]$ be a weak solution of Problem (O) satisfying the hypotheses of Theorem 3.1, and let $\left[u_{h}, \tau_{h}, p_{h}\right] \in X_{h}$ be a solution of

$$
\left\langle F_{h}\left(\left[u_{h}, \tau_{h}, p_{h}\right]\right),\left[v_{h}, \sigma_{h}, q_{h}\right]\right\rangle=0, \quad \forall\left[v_{h}, \sigma_{h}, q_{h}\right] \in Y_{h},
$$

where $F_{h}$ is defined in (4.35), which is sufficiently close to $\left[u_{0}, \tau_{0}, p_{0}\right]$ in the sense of Theorem 3.1 and also satisfies the hypotheses of Theorem 3.2. Then for some constants $c_{1}, \ldots, c_{5}$, the following a posteriori error estimates hold

$$
\begin{gathered}
\left\{\left\|u_{0}-u_{h}\right\|_{1,2}^{2}+\left\|\tau_{0}-\tau_{h}\right\|_{1,2}^{2}+\left\|p_{0}-p_{h}\right\|_{0,2}^{2}\right\}^{1 / 2} \leq \\
c_{1}\left\{\sum_{T} \eta_{T}^{2}\right\}^{1 / 2}+c_{2}\left\{\sum_{T}\left(\left\|u_{h}\right\|_{\infty, T} \delta\left(h_{T}, u_{h}\right)\right)^{2}\left\|R_{s}\right\|_{0,2 ; T}^{2}\right\}^{1 / 2}+c_{3}\left\{\sum_{T} h_{T}^{2}\left\|f-\pi_{k_{u}, T} f\right\|_{0,2 ; T}^{2}\right\}^{1 / 2},
\end{gathered}
$$


and

$$
\begin{aligned}
\eta_{T} \leq & c_{4}\left\{\left\|u_{0}-u_{h}\right\|_{1,2 ; w_{T}}^{2}+\left\|\tau_{0}-\tau_{h}\right\|_{1,2 ; w_{T}}^{2}+\left\|p_{0}-p_{h}\right\|_{0,2 ; w_{T}}^{2}\right\}^{1 / 2} \\
& +c_{5}\left\{\sum_{T^{\prime} \subset w_{T}} h_{T^{\prime}}^{2}\left\|f-\pi_{k_{u}, T^{\prime}} f\right\|_{0,2 ; T^{\prime}}^{2}\right\}^{1 / 2} .
\end{aligned}
$$

where $R_{s}$ and $\eta_{T}$ are as defined above.

Remark: The constants $c_{1}, \ldots, c_{5}$ in (4.65),(4.66) are independent of $h$ but depend upon the true solution $\left[u_{0}, \tau_{0}, p_{0}\right]$.

\section{Proof:}

Firstly we establish the existence of the derivative of $F$ and show that it is Lipschitz continuous in a neighbourhood of $\left[u_{0}, \tau_{0}, p_{0}\right]$.

Let $D F_{0} \in \mathcal{L}\left(X, Y^{*}\right)$ be defined by

$$
\begin{aligned}
& \left\langle D F_{0}([u, \tau, p]),[v, \sigma, q]\right\rangle:= \\
& \int_{\Omega}\left(\tau+\lambda\left((u \cdot \nabla) \tau_{0}+\left(u_{0} \cdot \nabla\right) \tau+\frac{\gamma}{\alpha}\left(\tau \tau_{0}+\tau_{0} \tau\right)+g_{a}\left(\tau, \nabla u_{0}\right)+g_{a}\left(\tau_{0}, \nabla u\right)\right)-2 \alpha \mathbf{D}(u)\right): \sigma d A \\
& \quad+\int_{\Omega} q \nabla \cdot u d A+\int_{\Omega}(2(1-\alpha) \mathbf{D}(u)-p \mathbb{I}+\tau): \nabla v d A
\end{aligned}
$$

for $[v, \sigma, q] \in Y$.

Now, using the continuous imbedding of $H^{1}$ in $L^{4}$,

$$
\begin{aligned}
& \left\langle F([u, \tau, p])-F\left(\left[u_{0}, \tau_{0}, p_{0}\right]\right)-D F_{0}\left(\left[u-u_{0}, \tau-\tau_{0}, p-p_{0}\right]\right),[v, \sigma, q]\right\rangle \\
& =\int_{\Omega} \lambda\left((u \cdot \nabla) \tau-(u \cdot \nabla) \tau_{0}-\left(u_{0} \cdot \nabla\right) \tau+\left(u_{0} \cdot \nabla\right) \tau_{0}+\frac{\gamma}{\alpha}\left(\tau \tau-\tau \tau_{0}-\tau_{0} \tau+\tau_{0} \tau_{0}\right)\right. \\
& \left.\quad \quad+g_{a}(\tau, \nabla u)-g_{a}\left(\tau, \nabla u_{0}\right)-g_{a}\left(\tau_{0}, \nabla u\right)+g_{a}\left(\tau_{0}, \nabla u_{0}\right)\right): \sigma d A \\
& =\int_{\Omega} \lambda\left(\left(\left(u-u_{0}\right) \cdot \nabla\right)\left(\tau-\tau_{0}\right)+\frac{\gamma}{\alpha}\left(\tau-\tau_{0}\right)\left(\tau-\tau_{0}\right)+g_{a}\left(\tau-\tau_{0}, \nabla\left(u-u_{0}\right)\right)\right): \sigma d A \\
& \leq c \lambda\left(\left\|u-u_{0}\right\|_{0,4}\left\|\nabla\left(\tau-\tau_{0}\right)\right\|_{0,2}+\frac{\gamma}{\alpha}\left\|\tau-\tau_{0}\right\|_{0,4}\left\|\tau-\tau_{0}\right\|_{0,2}\right. \\
& \left.\quad+4\left\|\tau-\tau_{0}\right\|_{0,4}\left\|\nabla\left(u-u_{0}\right)\right\|_{0,2}\right)\|\sigma\|_{0,4} \\
& \leq c \lambda\left(\left\|u-u_{0}\right\|_{1,2}\left\|\tau-\tau_{0}\right\|_{1,2}+\frac{\gamma}{\alpha}\left\|\tau-\tau_{0}\right\|_{1,2}^{2}+4\left\|\tau-\tau_{0}\right\|_{1,2}\left\|u-u_{0}\right\|_{1,2}\right)\|\sigma\|_{1,2} \\
& \leq c \lambda\left(\frac{\gamma}{\alpha}+5\right)\left\|[u, \tau, p]-\left[u_{0}, \tau_{0}, p_{0}\right]\right\|_{X}^{2}\|[v, \sigma, q]\|_{Y} .
\end{aligned}
$$

Thus, as

$$
\lim _{[u, \tau, p] \rightarrow\left[u_{0}, \tau_{0}, p_{0}\right]} \frac{\left\|F([u, \tau, p])-F\left(\left[u_{0}, \tau_{0}, p_{0}\right]\right)-D F_{0}\left(\left[u-u_{0}, \tau-\tau_{0}, p-p_{0}\right]\right)\right\|_{\mathcal{L}\left(X, Y^{*}\right)}}{\left\|[u, \tau, p]-\left[u_{0}, \tau_{0}, p_{0}\right]\right\|_{X}}=0,
$$


we have that $F$ is differentiable about $\left[u_{0}, \tau_{0}, p_{0}\right]$.

Next, to observe the Lipschitz continuity of the derivative, let $D F_{1}(\cdot)$ denote the derivative at $\left[u_{1}, \tau_{1}, p_{1}\right]$.

Then, for $[v, \sigma, q] \in Y,[u, \tau, p] \in B\left(\left[u_{0}, \tau_{0}, p_{0}\right], R_{0}\right)$,

$$
\begin{aligned}
& \left\langle D F_{1}([u, \tau, p])-D F_{0}([u, \tau, p]),[v, \sigma, q]\right\rangle \\
& \quad=\int_{\Omega} \lambda\left((u \cdot \nabla)\left(\tau_{1}-\tau_{0}\right)+\left(\left(u_{1}-u_{0}\right) \cdot \nabla\right) \tau+\frac{\gamma}{\alpha}\left(\tau\left(\tau_{1}-\tau_{0}\right)+\left(\tau_{1}-\tau_{0}\right) \tau\right)\right. \\
& \left.\quad+g_{a}\left(\tau, \nabla\left(u_{1}-u_{0}\right)\right)+g_{a}\left(\left(\tau_{1}-\tau_{0}\right), \nabla u\right)\right): \sigma d A \\
& \quad \leq 2 c \lambda\left(\frac{\gamma}{\alpha}+5\right)\left\|\left[u_{1}, \tau_{1}, p_{1}\right]-\left[u_{0}, \tau_{0}, p_{0}\right]\right\|\left\|_{X}\right\|[u, \tau, p]\|\|_{X}\|[v, \sigma, q]\|_{Y} .
\end{aligned}
$$

i.e,

$$
\begin{aligned}
\frac{\left\|D F_{1}([u, \tau, p])-D F_{0}([u, \tau, p])\right\|_{\mathcal{L}\left(X, Y^{*}\right)}}{\left\|\left[u_{1}, \tau_{1}, p_{1}\right]-\left[u_{0}, \tau_{0}, p_{0}\right]\right\| \|_{X}} & \leq 2 c\left(\frac{\gamma}{\alpha}+5\right)\|[u, \tau, p]\| \|_{X} \\
& \leq 2 c\left(\frac{\gamma}{\alpha}+5\right)\left(\left\|\left[u_{0}, \tau_{0}, p_{0}\right]\right\|_{X}+R_{0}\right)=\Upsilon
\end{aligned}
$$

Having verified the hypotheses, the stated results (4.65) and (4.66) now follow from (4.45),(4.47), (4.50),(4.51), and (4.64).

A stabilization of (2.4) using the Discontinuous Galerkin (DG) method modifies the definition of $F_{h}(\cdot)$ to

$$
\begin{array}{r}
\left\langle F_{h}\left(\left[u_{h}, \tau_{h}, p_{h}\right]\right),\left[v_{h}, \sigma_{h}, q_{h}\right]\right\rangle:=\left\langle F\left(\left[u_{h}, \tau_{h}, p_{h}\right]\right),\left[v_{h}, \sigma_{h}, q_{h}\right]\right\rangle \\
-\sum_{T} \int_{\partial T^{-}} \lambda u_{h} \cdot \mathbf{n}\left(\tau^{h+}-\tau^{h-}\right): \sigma_{h} d A, \\
\forall\left[v_{h}, \sigma_{h}, q_{h}\right] \in\left[M_{h}, \Sigma_{h}, Q_{h}\right] .
\end{array}
$$

where $\partial T^{-}$represents the inflow boundary of the simplex $T$ defined as

$$
\partial T^{-}:=\{x \in \partial T: u(x) \cdot \mathbf{n}(x)<0\} .
$$

Baranger and Sandri in [1] proved the existence of the solution to (4.72) for the Oldroyd-B model $(\gamma=0)$, and also established a priori error estimates for the approximation.

For the DG method, the consistency error is then

$$
\left\langle F\left(\left[u_{h}, \tau_{h}, p_{h}\right]\right)-F_{h}\left(\left[u_{h}, \tau_{h}, p_{h}\right]\right),\left[v_{h}, \sigma_{h}, q_{h}\right]\right\rangle=\sum_{T} \int_{\partial T^{-}} \lambda u_{h} \cdot \mathbf{n}\left(\tau^{h+}-\tau^{h-}\right): \sigma_{h} d A .
$$


Thus,

$$
\begin{aligned}
\left\|F\left(\left[u_{h}, \tau_{h}, p_{h}\right]\right)-F_{h}\left(\left[u_{h}, \tau_{h}, p_{h}\right]\right)\right\|_{Y_{h}^{*}} & \leq \sup _{\substack{\left[v_{h}, \sigma_{h}, q_{h}\right] \in Y_{h} \\
\left\|\left[v_{h}, \sigma_{h}, q_{h}\right]\right\|_{Y}=1}} \sum_{T}\left|\int_{\partial T^{-}} \lambda u_{h} \cdot \mathbf{n}\left(\tau^{h+}-\tau^{h-}\right): \sigma_{h} d A\right| \\
& \leq \sup _{\substack{\left.\left[v_{h}, \sigma_{h}, q_{h}\right] \in Y_{h} \\
\| v_{h}, \sigma_{h}, q_{h}\right] \|_{Y}=1}} \sum_{T}\left\|\lambda u_{h} \cdot \mathbf{n}\left(\tau^{h+}-\tau^{h-}\right)\right\|_{2 ; \partial T^{-}}\left\|\sigma_{h}\right\|_{2, \partial T^{-}} \\
& \leq \sup _{\substack{\left[v_{h}, \sigma_{h}, q_{h}\right] \in Y_{h} \\
\left\|\left[v_{h}, \sigma_{h}, q_{h}\right]\right\| \|_{Y}=1}} \sum_{T} c h_{T}^{-1 / 2}\left\|\lambda u_{h} \cdot \mathbf{n}\left(\tau^{h+}-\tau^{h-}\right)\right\|_{2, \partial T^{-}}\left\|\sigma_{h}\right\|_{0,2 ; T} \\
& \leq c^{*}\left\{\sum_{T} h_{T}^{-1}\left\|\lambda u_{h} \cdot \mathbf{n}\left(\tau^{h+}-\tau^{h-}\right)\right\|_{2 ; \partial T^{-}}^{2}\right\}^{1 / 2} .
\end{aligned}
$$

Using (4.74) instead of (4.50) we can then modify the result to obtain a similar estimate for the DG stabilization.

Theorem 4.2 Let $\left[u_{0}, \tau_{0}, p_{0}\right]$ be a weak solution of Problem (O) satisfying the hypotheses of Theorem 3.1, and let $\left[u_{h}, \tau_{h}, p_{h}\right] \in X_{h}$ be a solution of

$$
\left\langle F_{h}\left(\left[u_{h}, \tau_{h}, p_{h}\right]\right),\left[v_{h}, \sigma_{h}, q_{h}\right]\right\rangle=0, \quad \forall\left[v_{h}, \sigma_{h}, q_{h}\right] \in Y_{h},
$$

where $F_{h}$ is given in (4.35), which is sufficiently close to $\left[u_{0}, \tau_{0}, p_{0}\right]$ in the sense of Theorem 3.1 and also satisfies the hypotheses of Theorem 3.2. Then for some constants $c_{1}, \ldots, c_{5}$, the following a posteriori error estimates hold

$$
\begin{gathered}
\left\{\left\|u_{0}-u_{h}\right\|_{1,2}^{2}+\left\|\tau_{0}-\tau_{h}\right\|_{1,2}^{2}+\left\|p_{0}-p_{h}\right\|_{0,2}^{2}\right\}^{1 / 2} \leq \\
c_{1}\left\{\sum_{T} \eta_{T}^{2}\right\}^{1 / 2}+c_{2}\left\{\sum_{T} h_{T}^{-1}\left\|\lambda u_{h} \cdot \mathbf{n}\left(\tau^{h+}-\tau^{h-}\right)\right\|_{2, \partial T^{-}}^{2}\right\}^{1 / 2}+c_{3}\left\{\sum_{T} h_{T}^{2}\left\|f-\pi_{k_{u}, T} f\right\|_{0,2 ; T}^{2}\right\}^{1 / 2},
\end{gathered}
$$

and

$$
\begin{aligned}
\eta_{T} \leq & c_{4}\left\{\left\|u_{0}-u_{h}\right\|_{1,2 ; w_{T}}^{2}+\left\|\tau_{0}-\tau_{h}\right\|_{1,2 ; w_{T}}^{2}+\left\|p_{0}-p_{h}\right\|_{0,2 ; w_{T}}^{2}\right\}^{1 / 2} \\
& +c_{5}\left\{\sum_{T^{\prime} \subset w_{T}} h_{T^{\prime}}^{2}\left\|f-\pi_{k_{u}, T^{\prime}} f\right\|_{0,2 ; T^{\prime}}^{2}\right\}^{1 / 2} .
\end{aligned}
$$

where $\eta_{T}$ is as defined above.

\section{$5 \quad$ Numerical Experiments}

In this section, we present numerical experiments based on the a posteriori error estimate (4.65). 
Let $N$ represent the number of degrees of freedom associated with velocity, pressure and stress combined. For $x_{h}$ an approximation to $x$, we denote the $H^{1}$ and $L^{2}$ errors associated with $x_{h}$ as:

$$
E_{1}\left(x_{h}\right):=\left\|x-x_{h}\right\|_{1,2} \quad \text { and } \quad E_{0}\left(x_{h}\right):=\left\|x-x_{h}\right\|_{0,2} .
$$

Associated with the approximations to velocity, pressure and stress, we define two total error terms:

$$
E_{0}:=\left\{\left(E_{1}\left(u_{h}\right)\right)^{2}+\left(E_{0}\left(p_{h}\right)\right)^{2}+\left(E_{0}\left(\tau_{h}\right)\right)^{2}\right\}^{1 / 2}
$$

and

$$
E_{1}:=\left\{\left(E_{1}\left(u_{h}\right)\right)^{2}+\left(E_{0}\left(p_{h}\right)\right)^{2}+\left(E_{1}\left(\tau_{h}\right)\right)^{2}\right\}^{1 / 2} .
$$

For $E_{0}$ the error in the stress is measured in the $L^{2}$ norm, whereas for $E_{1}$ the error in the stress is measured in the $H^{1}$ norm. We define the error indicator $\widetilde{E}$, as the right hand side of (4.65) with the constants $c_{1}=1$ and $c_{2}=c_{3}=0$. Note that the second term on the right hand side of (4.65) can be bounded by the first term, thus the second term can be written as a constant multiple of the first term. Note also, that the third term on the right hand side of (4.65) is a higher order term and may in general be ignored. Next, we define two effectivity indices, $I_{\text {eff }}$ and $I_{\text {eff }}$ as:

$$
I_{e f f_{0}}:=\frac{\widetilde{E}}{E_{0}} \quad \text { and, } \quad I_{e f f_{1}}:=\frac{\widetilde{E}}{E_{1}} .
$$

For the numerical computations we used as the approximation spaces for the velocity and pressure the Taylor-Hood pair; continuous piecewise quadratics and continuous piecewise linears, respectively. For the polymetric stress tensor the approximation space was comprised of continuous piecewise linear elements. The approximating nonlinear system of equations was solved by lagging the nonlinearities and then iteratively solving the resulting linear system to steady state. The GMRES and BiCGSTAB algorithms, with ILU preconditioning, were used to solve the linear systems.

A standard adaptive computational algorithm was used in the computations:

1. Construct an initial coarse mesh $\Pi_{h, j}, j=0$.

2. Compute the approximate solution on $\Pi_{h, j}$.

3. Compute the global a posteriori error estimate. If the estimate is less than a preset tolerance exit the computation.

4. Based upon each triangles' contribution to the global error estimate, determine which triangles are to be refined.

5. Refine the mesh and set $j=j+1$. Return to step 2 .

We used a 15-15 rule for determining which triangles were to be refined. For a triangle to be a candidate for refinement its contribution to the global a posteriori error must have been at least $15 \%$ 
of that of the element with the largest contribution. In addition, the maximum number of triangles designated for refinement at each iteration was limited to $15 \%$ of the total number of triangles. Thus, a triangle was refined if it was one of the $15 \%$ "worst" triangles and its contribution to the global a posteriori error estimate was at least $15 \%$ of that of the "very worst" triangle. The local refinement algorithm used is described in [18].

The first two examples, taken from [11], have singularities just exterior to their domains, near the points $(2,2)$ and $(0,0)$ respectively. In order to have a known solution for the polymetric stress $\tau$, a right hand side function is added to (2.4). This function is then calculated so that the solution for $\tau$ is $\tau=2 \alpha \mathbf{D}(u)$. For each example computations with $\lambda=0.1, \lambda=0.5$ and $\lambda=1.0$ were performed. In all the computations we have used $\alpha=0.41$, corresponding to the value for the MIT Boger fluid $[25]$.

\section{Example 1.}

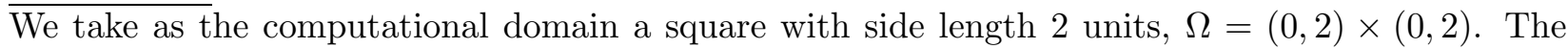
velocity, polymetric stress, and pressure used are

$$
u(x, y):=\left[\begin{array}{c}
-(4.1-x-y)^{-1 / 3} \\
(4.1-x-y)^{-1 / 3}
\end{array}\right], \quad \tau(x, y):=2 \alpha \mathbf{D}(u), \quad p(x, y):=x+y .
$$

\section{Example 2.}

For this example $\Omega$ is an L-shaped domain given by $\Omega=(-1,1) \times(-1,1)-(0,1) \times(0,1)$. The velocity, polymetric stress, and pressure used are

$$
u(x, y):=\left[\begin{array}{c}
\frac{(y-0.1)}{\left[(x-0.1)^{2}+(y-0.1)^{2}\right]^{1 / 2}} \\
\frac{(0.1-x)}{\left[(x-0.1)^{2}+(y-0.1)^{2}\right]^{1 / 2}}
\end{array}\right], \quad \tau:=2 \alpha \mathbf{D}(u), \quad p(x, y):=(2-x-y)^{1 / 2} .
$$

The numerical results for Example $1(\lambda=0.5)$ are presented in Tables 5.1 and 5.2 , which contain for each grid used the total degrees of freedom $N$, the exact errors associated with the velocity, pressure and polymetric stress $\left(L^{2}\right.$ and $\left.H^{1}\right)$, the total error terms $E_{0}, E_{1}$, the error indicator $\widetilde{E}$, and the effectivity indices $I_{e f f_{0}}$ and $I_{e f f_{1}}$. The adaptive grids generated for Examples 1 and $2(\lambda=0.5)$ are displayed in Figure 5.1.

From Tables 5.1 and 5.2 we observe that $\widetilde{E}$ is a more robust predictor for $E_{0}$ than it is for $E_{1}$ indicated by the fact that $I_{\text {eff }}$ is more consistent than $I_{e f f_{1}}$ under the refinement process. (It is worth noting that $\widetilde{E}$ was computed with the values $c_{1}=c_{3}=1$ and $c_{2}=0$. More appropriate choices are currently under investigation.) Presented in Table 5.2 are numerical computations for Example 1 with $\lambda=0.5$ for a uniformly refined mesh. Successive entries in Table 5.2 correspond to "half-refinements" of the current mesh. By considering alternate entries in Table 5.2 we observe optimal orders of approximation for the velocity, stress and pressure. Namely, $O\left(h^{2}\right)$ in $\|\cdot\|_{H^{1}}$, $O\left(h^{2}\right)$ in $\|\cdot\|_{L^{2}}$ and $O\left(h^{2}\right)$ in $\|\cdot\|_{L^{2}}$, respectively.

\section{Remarks:}

1. Note that $E_{0} \leq E_{1}$, so the upper bound (4.65) is also a valid estimator for $E_{0}$. 


\begin{tabular}{|c|c|cc|c|cc|c|cc|}
\hline$N$ & $E_{1}\left(u_{h}\right)$ & $E_{0}\left(\tau_{h}\right)$ & $E_{1}\left(\tau_{h}\right)$ & $E_{0}\left(p_{h}\right)$ & $E_{0}$ & $E_{1}$ & $\widetilde{E}$ & $I_{\text {eff }}$ & $I_{\text {eff }}$ \\
\hline 2946 & 0.13594 & 0.17597 & 4.46413 & 0.05164 & 0.22828 & 4.46650 & 1.35073 & 5.91 & 0.302 \\
3378 & 0.05492 & 0.08871 & 2.84009 & 0.03016 & 0.10861 & 2.84078 & 0.54983 & 5.06 & 0.193 \\
3908 & 0.04232 & 0.04810 & 2.67561 & 0.01457 & 0.06570 & 2.67598 & 0.42855 & 6.52 & 0.160 \\
4506 & 0.01500 & 0.02235 & 1.46776 & 0.00770 & 0.02800 & 1.46786 & 0.15169 & 5.41 & 0.103 \\
5226 & 0.01085 & 0.01131 & 1.36705 & 0.00338 & 0.01604 & 1.36709 & 0.11271 & 7.02 & 0.082 \\
6070 & 0.00394 & 0.00543 & 0.71784 & 0.00194 & 0.00699 & 0.71786 & 0.04008 & 5.73 & 0.055 \\
7102 & 0.00282 & 0.00288 & 0.67319 & 0.00093 & 0.00414 & 0.67320 & 0.02925 & 7.05 & 0.043 \\
8184 & 0.00146 & 0.00173 & 0.37441 & 0.00069 & 0.00237 & 0.37441 & 0.01469 & 6.18 & 0.039 \\
9626 & 0.00113 & 0.00120 & 0.35306 & 0.00075 & 0.00181 & 0.35306 & 0.01155 & 6.35 & 0.032 \\
11230 & 0.00090 & 0.00096 & 0.24833 & 0.00069 & 0.00149 & 0.24833 & 0.00901 & 6.03 & 0.036 \\
13270 & 0.00075 & 0.00079 & 0.23056 & 0.00059 & 0.00124 & 0.23057 & 0.00756 & 6.06 & 0.032 \\
15710 & 0.00062 & 0.00066 & 0.21090 & 0.00045 & 0.00101 & 0.21090 & 0.00630 & 6.19 & 0.029 \\
\hline
\end{tabular}

Table 5.1: Exact and approximate errors, and effectivity indices for Example 1 using adaptive refinements for $\lambda=0.5$

\begin{tabular}{|c|c|cc|c|cc|c|cc|}
\hline$N$ & $E_{1}\left(u_{h}\right)$ & $E_{0}\left(\tau_{h}\right)$ & $E_{1}\left(\tau_{h}\right)$ & $E_{0}\left(p_{h}\right)$ & $E_{0}$ & $E_{1}$ & $\widetilde{E}$ & $I_{\text {eff }}$ & $I_{\text {eff }}$ \\
\hline 2946 & 0.13594 & 0.17597 & 4.46413 & 0.05164 & 0.22828 & 4.46650 & 1.35073 & 5.91 & 0.302 \\
5646 & 0.05492 & 0.08871 & 2.83993 & 0.03017 & 0.10861 & 2.84063 & 0.54974 & 5.06 & 0.193 \\
11286 & 0.04231 & 0.04809 & 2.67556 & 0.01452 & 0.06568 & 2.67594 & 0.42842 & 6.52 & 0.160 \\
22086 & 0.01495 & 0.02231 & 1.46649 & 0.00770 & 0.02794 & 1.46658 & 0.15119 & 5.41 & 0.103 \\
44166 & 0.01079 & 0.01125 & 1.36589 & 0.00336 & 0.01595 & 1.36593 & 0.11205 & 7.02 & 0.082 \\
87366 & 0.00371 & 0.00529 & 0.71163 & 0.00190 & 0.00674 & 0.71165 & 0.03801 & 5.63 & 0.053 \\
\hline
\end{tabular}

Table 5.2: Exact and approximate errors, and effectivity indices for Example 1 using uniform refinements for $\lambda=0.5$.

2. Notice that $E_{1}$ is completely dominated by the error in the polymetric stress(measured in the $H^{1}$ norm). On the other hand, the magnitude of the components that comprise $E_{0}$ are all consistent and none of them dominate the true error as the polymetric stress does in $E_{1}$.

We have only presented results for Example 1 with $\lambda=0.5$. Our other computations for Examples 1 and 2 , with different values of $\lambda$ between 0.1 and 1.0, follow the same pattern.

\section{Example 3.}

For Example 3 we consider a benchmark problem in viscoelastic fluid flow simulation; channel flow with a cylindrical obstacle [4]. The ratio of the channel height to the cylinder diameter, $\mathrm{H}$, is taken to be 4 , while the maximum inflow velocity is set at 1.5. The boundary conditions imposed are as follows. For velocity: a fully developed flow field (parabolic profile) at the inflow and outflow boundaries, and a non-slip $(u=0)$ condition along the other boundaries. For polymetric stress: along the inflow boundary the polymetric stress for a fully developed channel flow field satisfying the Oldroyd-B constitutive law is prescribed. For pressure: the pressure is fixed at one of the inflow mesh points to zero.

The numerical computations for Example 3 are summarized in Tables 5.3 and 5.4, and Figures 5.2 and 5.3. The adaptive grids generated are presented in Figure 5.2. For comparison, computations 


\begin{tabular}{|cc|cc|}
\hline$N_{A R}$ & $\widetilde{E}_{A R}$ & $N_{U R}$ & $\widetilde{E}_{U R}$ \\
\hline 4832 & 4.2811 & 4832 & 4.2811 \\
5672 & 2.7743 & 9248 & 2.4274 \\
6828 & 2.2855 & 18496 & 1.7297 \\
8178 & 1.2780 & 36160 & 0.7476 \\
10100 & 0.9523 & 72320 & 0.5346 \\
12600 & 0.7288 & 142976 & 0.2183 \\
15488 & 0.5636 & - & - \\
18824 & 0.4526 & - & - \\
22900 & 0.3624 & - & - \\
27840 & 0.2829 & - & - \\
34012 & 0.2220 & - & - \\
41448 & 0.1843 & - & - \\
\hline
\end{tabular}

Table 5.3: Degrees of freedom and approximate errors for the channel flow problem for $\lambda=0.1$. Adaptive refinements (AR) on the left and uniform refinements (UR) on the right.

on uniform meshes are also presented in Tables 5.3 and 5.4. Successive entries under uniform refinements correspond to "half-refinements" of the current mesh. (Thus, for comparison with uniform refinements of the initial mesh, alternate entries in the table need to be ignored.) From the adaptively generated meshes, Figure 5.2, it is clear that the refinement procedure correctly refines the triangles in the regions of difficulty which are known to exists for this particular flow.

For Example 3, the

$$
\frac{\text { Error }}{\text { Unknown }} \text { reduction factor }:=\frac{\text { Initial Error/Final Error }}{\text { Final Number of Unknowns/Initial Number of Unknowns }} \text {, }
$$

was for the case $\lambda=0.1,2.70$ for adaptive refinements compared with 0.66 for uniform refinements. For $\lambda=0.5$ these factors were 2.66 and 0.54 respectively. The savings obtained in using an adaptive strategy is clearly significant, especially for problems in viscoelasticity where, to obtain an accurate approximation, a large number of unknowns is required. 


\begin{tabular}{|cc|cc|}
\hline$N_{A R}$ & $\widetilde{E}_{A R}$ & $N_{U R}$ & $\widetilde{E}_{U R}$ \\
\hline \hline 4832 & 4.6662 & 4832 & 4.6662 \\
5768 & 2.9771 & 9248 & 2.7106 \\
6932 & 2.2408 & 18496 & 1.8578 \\
8316 & 1.4124 & 36160 & 0.9540 \\
10264 & 1.0594 & 72320 & 0.5868 \\
12472 & 0.7792 & 142976 & 0.2903 \\
15220 & 0.6349 & - & - \\
18692 & 0.5046 & - & - \\
22852 & 0.4087 & - & - \\
27524 & 0.3211 & - & - \\
33660 & 0.2535 & - & - \\
41088 & 0.2059 & - & - \\
\hline
\end{tabular}

Table 5.4: Degrees of freedom and approximate errors for the channel flow problem for $\lambda=0.5$. Adaptive refinements (AR) on the left and uniform refinements (UR) on the right. 


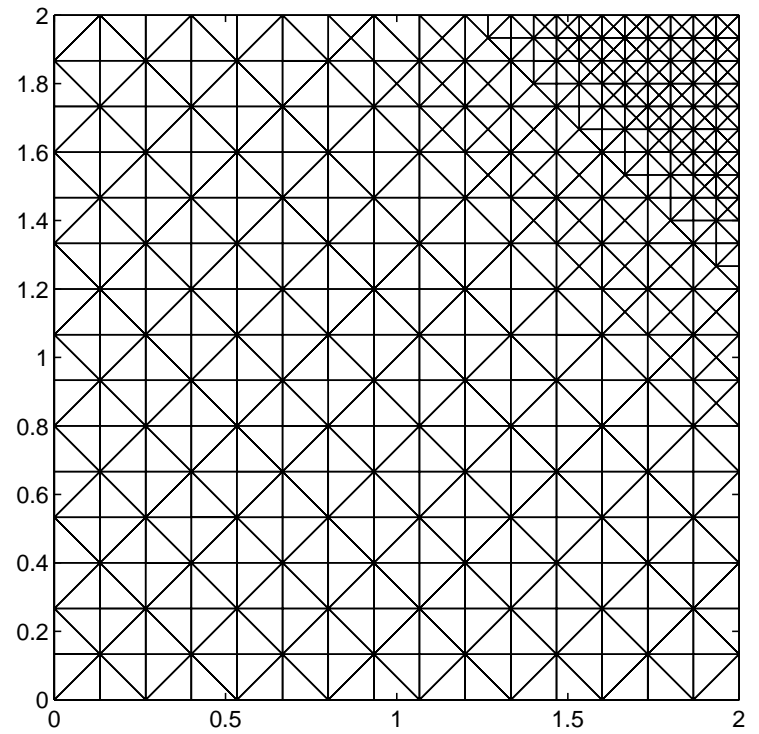

(a) 3 levels of refinement(Example 1, $\lambda=0.5$ ).

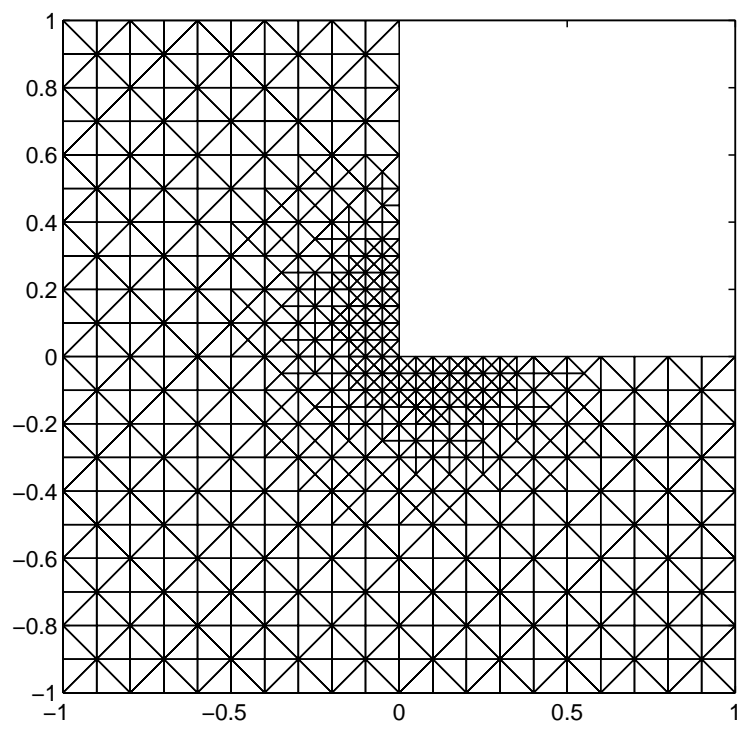

(c) 3 levels of refinement(Example 2, $\lambda=0.5$ ).

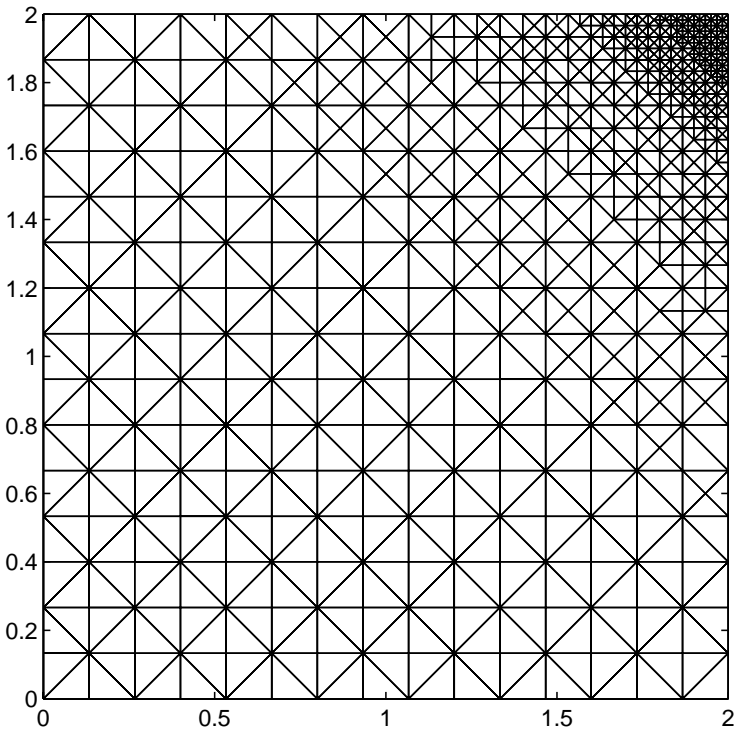

(b) 6 levels of refinement(Example 1, $\lambda=0.5$ ).

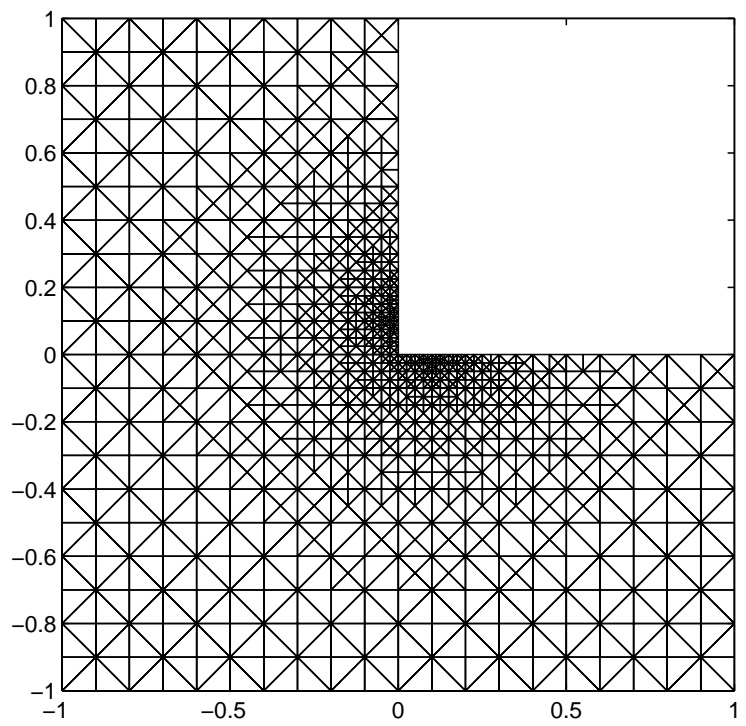

(d) 6 levels of refinement(Example 2, $\lambda=0.5$ ).

Figure 5.1: Adaptive meshes generated after three and six levels of refinement for Examples 1 and 2 with $\lambda=0.5$. 


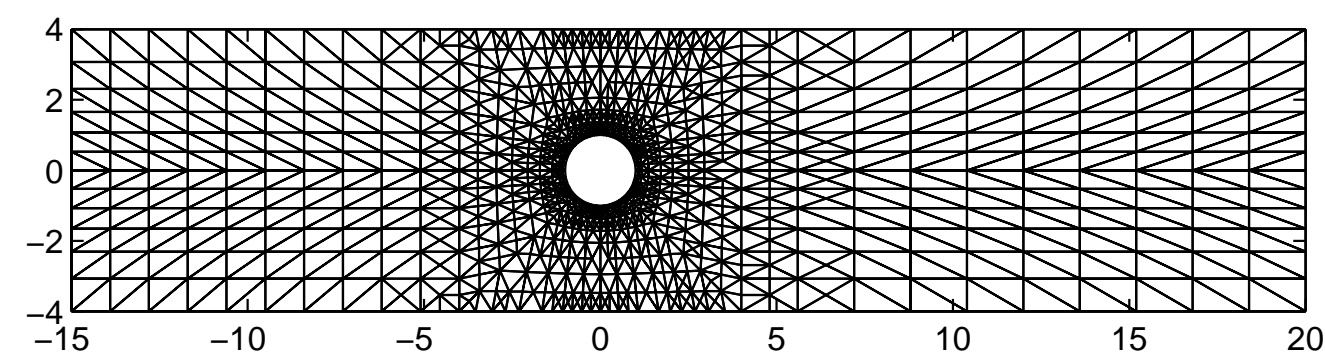

(a) 6 levels of refinement $(\lambda=0.1)$

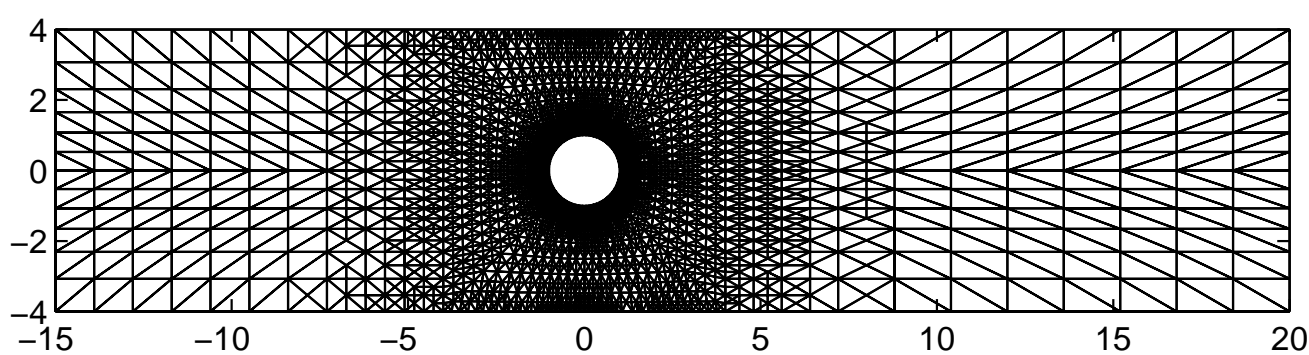

(b) 12 levels of refinement $(\lambda=0.1)$

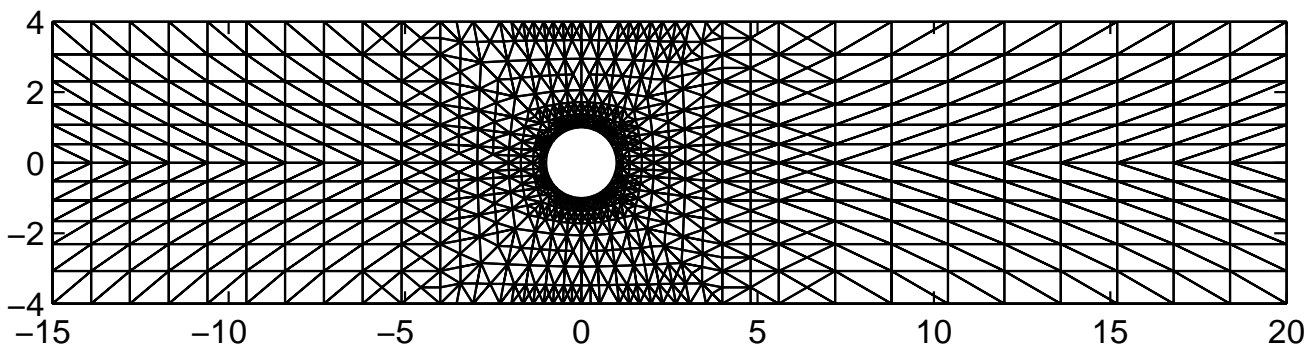

(c) 6 levels of refinement $(\lambda=0.5)$

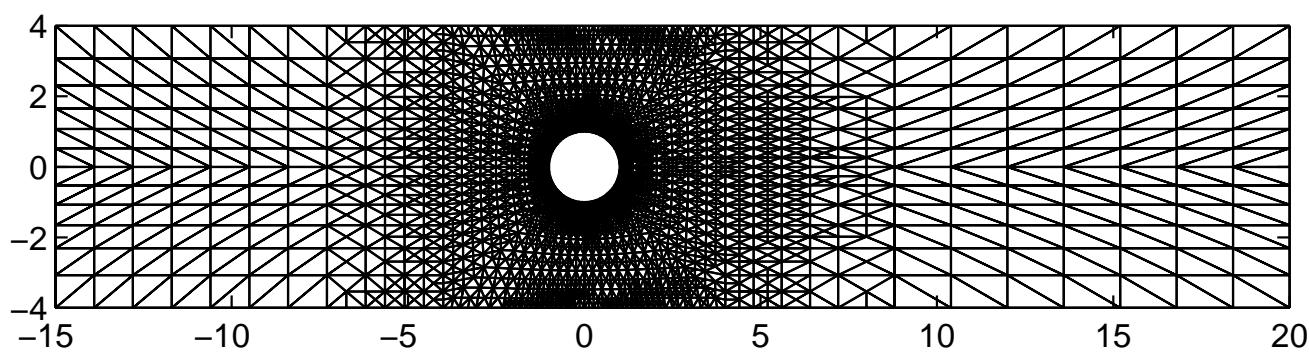

(d) 12 levels of refinement $(\lambda=0.5)$

Figure 5.2: Adaptive meshes generated for the channel flow problem for $\lambda=0.1$ and $\lambda=0.5$. 


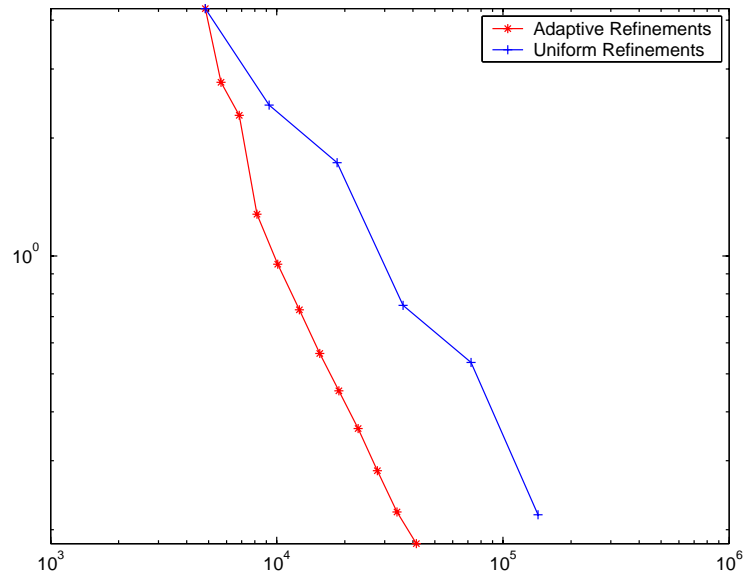

(a)

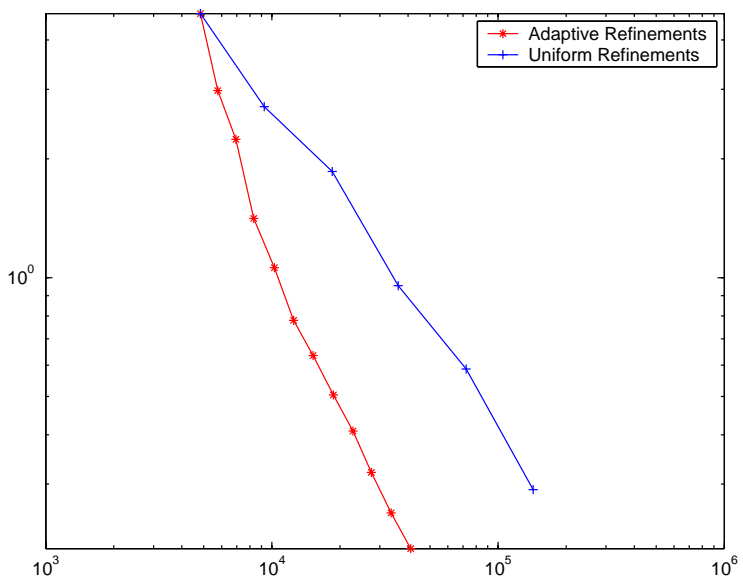

(b)

Figure 5.3: Log-Log plot of estimated error as a function of the degrees of freedom for the channel flow problem for (a) $\lambda=0.1$ and (b) $\lambda=0.5$. 


\section{References}

[1] J. Baranger and D. Sandri. Finite element approximation of viscoelastic fluid flow: Existence of approximate solutions and error bounds. I. Discontinuous constraints. Numer. Math., 63: 13-27, 1992.

[2] R.B. Bird, R.C. Armstrong, and O. Hassager. Dynamics of Polymeric Liquids. John Wiley and Sons, Inc., 1987.

[3] A.N. Brooks and T.J.R. Hughes. Streamline upwind/Petrov-Galerkin formulations for the convection dominated flows with particular emphasis on the incompressible Navier-Stokes equations. Comput. Methods. Appl. Mech. Engrg., 32:109-259, 1982.

[4] B. Caswell. Report on the IX ${ }^{t h}$ International workshop on numerical methods in non-Newtonian flows. J. Non-Newtonian Fluid Mech., 62:99-110, 1996.

[5] C. Chauvière and R.G. Owens. How accurate is your solution? Error indicators for viscoelastic flows calculations. J. Non-Newtonian Fluid Mech., 95:1-33, 2000.

[6] Ph. Clément. Approximation by finite elements using local regularization. RAIRO Anal. Numér., 2:77-84, 1975.

[7] K. Eriksson and C. Johnson. Adaptive streamline diffusion finite element methods for stationary convection-diffusion problems. Math. Comp., 60:167-188, 1993.

[8] V.J. Ervin and W.J. Layton. A study of defect correction finite difference methods for convection dominated convection diffusion equations. SIAM J. Numer. Anal., 26:169-179, 1989.

[9] V.J. Ervin, W.J. Layton, and J.M. Maubach. An adaptive defect correction method for viscous incompressible flow problems. SIAM J. Numer. Anal., 37:1165-1185, 2000.

[10] E. Fernandez-Cara, F. Guillen, and R.R. Ortega. Mathematical modeling and analysis of viscoelastic fluids of the oldroyd kind. In P.G. Ciarlet and J.L. Lions, editors, Handbook of numerical analysis, volume VIII, pages 543-661. Elsevier, Amsterdam, London, New York, Oxford, Paris, Shannon, Tokyo, 2002.

[11] G.N. Gatica, M. Gonzalez, and S. Meddahi. A low-order mixed finite element method for a class of quasi-Newtonian stokes flows. Preprint 02-01, Department of Mathematics, University of Concepcion, (available at http://www.ing-mat.udec.cl/investigacion/publicaciones/paginaspp/pp02-01.html), 2002.

[12] M.D. Gunzburger. Finite Element Methods for Viscous Incompressible Flows. Academic Press, 1989.

[13] C. Johnson, U. Navert, and J. Pitkaranta. Finite element methods for linear hyperbolic problems. Comput. Methods. Appl. Mech. Engrg., 45:285-312, 1984.

[14] R.C. King, M.R. Apelian, R.C. Armstrong, and R.A. Brown. Numerically stable finite element techniques for viscoelastic flow calculations in smooth and singular geometries. J. NonNewtonian Fluid Mech., 29:147-216, 1989. 
[15] C. Lasser and A. Toselli. An overlapping domain decomposition preconditioner for a class of discontinuous Galerkin approximations of advection-diffusion problems. To appear in Math. Comp. (available at http://www.sam.math.ethz.ch/ ${ }^{\sim}$ toselli/pub.html), 2000.

[16] P. Lesaint and P.A. Raviart. On a finite element method for solving the neutron transport equation, in: $C$ de Borr(Ed.) Mathematical Aspects of Finite Elements in Partial Differential Equations. Academic Press, New York, 1974.

[17] A.W. Liu, D.E. Bornside, R.C. Armstrong, and R.A. Brown. Viscoelastic flow of polymer solutions around a periodic, linear array of cylinders: Comparisons of predictions for microstructure and flow fields. J. Non-Newtonian Fluid Mech., 77:153-190, 1998.

[18] J. Maubach. Local bisection refinement for simplical grids generated by reflection. SIAM J. Sci. Comput., 16:210-227, 1995.

[19] R.G. Owens. A posteriori error estimates for spectral element solutions to viscoelastic flow problems. Comput. Methods. Appl. Mech. Engrg., 164:375-395, 1998.

[20] M. Picasso and J. Rappaz. Existence, a priori and a posteriori error estimates for a nonlinear three field problem arising from Oldroyd-B viscoelastic flows. Math. Model. Numer. Anal., 35(5):879-897, 2001.

[21] A. Quarteroni and A. Valli. Numerical Approximation of Partial Differential Equations. Springer-Verlag, 1994.

[22] D. Rajagopalan, R.C. Armstrong, and R.A. Brown. Finite element methods for calculation of steady state, viscoelastic flows using constitutive equations with a Newtonian viscosity. $J$. Non-Newtonian Fluid Mech., 36:159-192, 1990.

[23] D. Sandri. Finite element approximation of viscoelastic fluid flow: Existence of approximate solutions and error bounds. Continuous approximation of the stress. SIAM J. Numer. Anal., 31:362-377, 1994.

[24] J. Sun, N. Phan-Thien, and R.I. Tanner. An adaptive viscoelastic stress splitting scheme and its applications: AVSS/SI and AVSS/SUPG. J. Non-Newtonian Fluid Mech., 65:75-91, 1996.

[25] J. Sun, M.D. Smith, R.C. Armstrong, and R.A. Brown. Finite element method for viscoelastic flows based on discrete adaptive stress splitting and the discontinuous Galerkin method: DAVSS-G/DG. J. Non-Newtonian Fluid Mech., 86:281-307, 1999.

[26] M.J. Szady, T.R. Salomon, A.W. Liu, D.E. Bornside, R.C. Armstrong, and R.A. Brown. A new mixed finite element method for viscoelastic flows governed by differential constitutive equations. J. Non-Newtonian Fluid Mech., 59:215-243, 1995.

[27] R. Verfürth. A review of a posteriori error estimation and adaptive mesh refinement techniques. Wiley, Teubner, 1996. 